\title{
Evaluation of groundwater and surface water quality suitability for drinking and agricultural purposes in Kombolcha town area, eastern Amhara region, Ethiopia
}

\author{
Berihu Abadi Berhe ${ }^{1}$
}

Received: 25 July 2019 / Accepted: 24 April 2020 / Published online: 8 May 2020

(c) The Author(s) 2020

\begin{abstract}
The appropriateness of groundwater and surface water for domestic and agricultural uses was assessed in Kombolcha town located in Amhara region using water quality indexes. The town is one of the fast developing and emerging as a leading industrial town in Ethiopia and is selected as an industrial zone by the government. A total of eighteen groundwater and five surface water samples were collected using 250-ml sampling bottles at selected points and analyzed for major ions $\left(\mathrm{Ca}^{2+}\right.$, $\mathrm{Mg}^{2+}, \mathrm{Na}^{+}, \mathrm{K}^{+}, \mathrm{HCO}_{3}{ }^{-}, \mathrm{SO}_{4}{ }^{2-}, \mathrm{Cl}^{-}$) in the dry season (May 2017) and wet season (Nov, 2017). A water quality index (WQI) method was applied to evaluate the suitability of the groundwater for drinking purposes using eighteen groundwater sampling points and fourteen parameters (EC, TDS, $\mathrm{HCO}_{3}{ }^{-}, \mathrm{Ca}^{2+}, \mathrm{Mg}^{2+}, \mathrm{Na}^{+}, \mathrm{K}^{+}, \mathrm{Cl}, \mathrm{SO}_{4}{ }^{2-}, \mathrm{NO}_{3}{ }^{-}, \mathrm{PO}_{4}{ }^{3-}, \mathrm{Cu}, \mathrm{Fe}$ and $\mathrm{F}^{-}$), and each parameters were also compared with WHO drinking guidelines. According to WQI, groundwater samples of the present study show excellent $(72.2 \%)$ and good (27.8\%) in the dry season and excellent (83.3\%) and good (16.7\%) in the wet season. Assessment of groundwater samples from $\mathrm{Na}^{+}, \mathrm{Mg}^{2+}, \mathrm{K}^{+}, \mathrm{Cl}^{-}, \mathrm{SO}_{4}{ }^{2-}, \mathrm{HCO}_{3}{ }^{-}, \mathrm{NO}_{3}{ }^{-}, \mathrm{PO}_{4}{ }^{3-}, \mathrm{F}^{-}$and $\mathrm{Cu}$ parameters indicates that groundwater of the study area is chemically suitable for drinking purposes. However, some are unsuitable according to the EC, TDS and $\mathrm{Ca}^{2+}$ and all are not suitable with reference to iron (Fe). Groundwater and surface water quality for irrigation were evaluated using SAR, RSC, MR, PI, KI and EC. The results show that most of the groundwater and surface water samples were suitable/excellent for irrigation with some places in the study locations that belong to the good and permissible. The sodium hazard versus salinity hazard of the entire water sample collected in two seasons falls into category C2-S1 and C3-S1, indicating low alkali hazards and excellent irrigation water. Groundwater samples in wet seasons are more suitable than in the dry season for drinking and irrigation purposes, and groundwater samples are less polluted than surface water. The main source of pollution in the study area is the effluents from industries.
\end{abstract}

Keywords Water quality index $\cdot$ Drinking water $\cdot$ Irrigation water $\cdot$ Kombolcha

\section{Introduction}

Hydrochemistry is the primary determinant of domestic, irrigation or industrial water use. Groundwater quality is regulated by various variables, including climate, soil characteristics, lithology type, region topography and on-site human operations (Rajesh et al. 2002; Das Brijraj and Kaur 2007; Cloutier et al. 2008). Surface water and groundwater play

Berihu Abadi Berhe

berihu.abadi@mu.edu.et; berhag2000@gmail.com

1 Department of Geology, School of Earth Science, College of Natural and Computational Science, Mekelle University, 231 Mekelle, Ethiopia a significant role in offering drinking, irrigation and industrial water supply, with approximately 2.5 billion individuals worldwide depending on it (Connor 2015). The quantity and quality issues present a challenge to groundwater management since groundwater is a complement to surface water resources (Van der Gun 2012). The concept of the water quality index (WQI) was firstly used by Horton (1965) and was further developed by Brown et al. (1970).

Water quality index is a rating reflecting the collective impact of different water quality parameters on the whole quality of water, and it is accepted that reasonable evaluation based on single parameter cannot deliver the overall water quality and has been broadly utilized all over the world (Deininger and Maciunas 1971; Tiwari and Manzoor 1988; Li et al. 2010; Pati et al. 2014; Varol and Davraz 2015). Thus, 
a comprehensive appraisal of overall water quality using this method is exceptionally imperative.

The chemistry of surface water and groundwater is the main issue determinant water use for domestic, irrigation or industrial functions. Generally, the quality of groundwater is governed by a variety of variables, including climate, soil characteristics, types of lithology, the topography of the area and of course the human activities on the ground (Rajesh et al. 2002; Das Brijraj and Kaur 2007; Cloutier et al. 2008).

Currently, the rapid population increase, the use of agricultural fertilizers and the growth of industrializations and the disposal of industrial waste have all played a significant part in the contamination of surface water and groundwater contamination and have greatly boosted the stress on water quality (Chandra et al. 2015; Verma et al. 2016).

Nowadays, almost in all parts of Ethiopia, the demand for water and consecutive abstraction for groundwater has increased from time to time. This is because surface water bodies are susceptible to pollution and also due to population growth at an alarming rate, thus affecting the resource quality and thus the elevated anticipated cost of the use of contaminated surface water resources. Understanding the water quality used for drinking and irrigation and its potential adverse effects on human life and plant development is very essential to prevent problems and optimize the general public's health and crop output.

Kombolcha is one of the fast developing and emerging as a leading industrial town in Ethiopia, and it is one of the towns selected as an industrial zone by the government. The town is one of Ethiopia's towns with comparatively more large-scale manufacturing plants than the size of the town. The town's major existing industries are Steel Product Industry, Flour Factory, Textile Factory, ELFORA-Meat Processing Factory, BGI-Brewery Factory and Tannery. In this town, industrial wastewater mixed with river water is often used for irrigation industrial wastewaters containing toxic organic and inorganic chemicals that can be taken up by the crops and vegetables (Mohammed 2015).

In Ethiopia, from the increasing human population, uncontrolled urbanization and inadequate sanitation infrastructure cause serious quality degradation of surface waters. Hence, discharging of untreated industrial and municipal wastes to surface water increases with increasing urbanization (Hamere and Eyasu 2017). The quality of groundwater is poorly understood in the Kombolcha town, the eastern part of Amhara region, Ethiopia, whereas it is the only source of drinking and industrial purposes. A detailed study has not been carried out earlier for its suitability for drinking and irrigation purposes and the effect of factory effluents on the surface waters and groundwater has not been investigated well.

Therefore, the current study aims to carry out a comprehensive appraisal of the quality of groundwater resources using the water quality index (WQI) technique to assess its suitableness for drinking and the irrigation water quality of surface water and groundwater within the study was also evaluated by typical water quality index parameters.

\section{Study area}

It is nearby $23 \mathrm{~km}$ from Dessie town and $375 \mathrm{~km}$ from state capital Addis Ababa toward North along the road to Mekelle, which covers an area of about $120 \mathrm{~km}^{2}$. The groundwater wells were opened in the alluvial plain where it covers about $46 \%\left(55 \mathrm{~km}^{2}\right)$ of the study area. The study area is bounded between 571,500 and 583,800 mE longitude and 1,219,000 and 1,234,600 mN latitude (Fig. 1). It is located on the western margin of the Main Ethiopian Rift (MER). The MER is located at the northern termination of the East African Rift System (EARS) and extends from the Afar triple junction in the north to the Turkana Rift in the south (Fig. 1).

Small trees mostly acacia and thorny bushes are common; however, scarce eucalyptus trees are seen along the western in the ridges. Most of the area on the flat topography is used for agricultural farming by local people. June to September is the wet season, while the dry season extends from November to May.

The study area's mean annual rainfall is $1072.15 \mathrm{~mm}$ with the highest rainfall in July and August. The mean annual minimum and maximum temperature of the study area is $12.44{ }^{\circ} \mathrm{C}$ and $26.87^{\circ} \mathrm{C}$, respectively, with the mean annual temperature of the area that is $19.66{ }^{\circ} \mathrm{C}$.

The general physiographic map of the study area was prepared from DEM of the study area and is shown in Fig. 1. It is characterized by marked topographic variations and has a very high rugged topography. The altitude ranges from 1759 m.a.s.l on the flat areas, where the Kombolcha town is situated, to 2910 m.a.s.l at Yegof Mountain. The drainage network in this area is almost north-south direction, and major streams in this physiographic area include Borkena River, Eyole and Werka streams (Figs. 1 and 2).

\section{Geology and hydrogeology}

The geology of the study area is constituted by the rocks ranging in age from Eocene-Oligocene to Recent or Quaternary deposits. Stratigraphically, from bottom to top, they are Ashangie basalts (Eocene-Oligocene), Dessie basalt Formation and Ancharo Rhyolitic ignimbrite (Oligo-Miocene) and Quaternary sediments (GSE 2010) (Fig. 2).

The Ashangie basalts are also exposed as a faulted block along NW-SE trending escarpment. They are found as continuous and patchy outcrops with a sheet and blocky forms (Mengesha et al. 1996; Tesfaye et al. 2010). The Ashangie basalt is exposed along road cuts, stream beds, gentle and steep slopes of undulating mountain chains and low-lying 


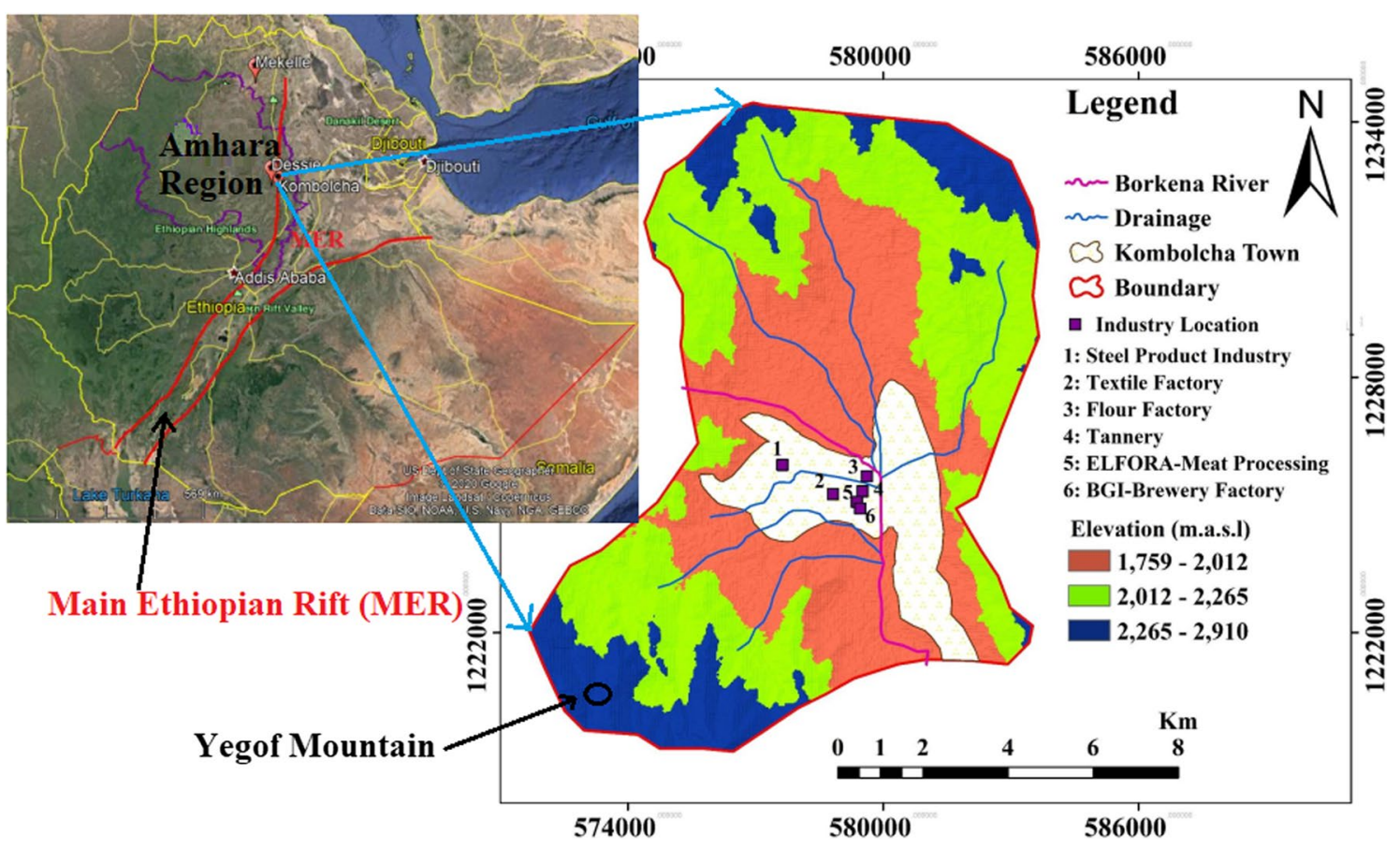

Fig. 1 Location map of the study area

Fig. 2 Geological map (modified after Geological Survey of Ethiopia GSE (2010) and water sampling point's map of the study area)

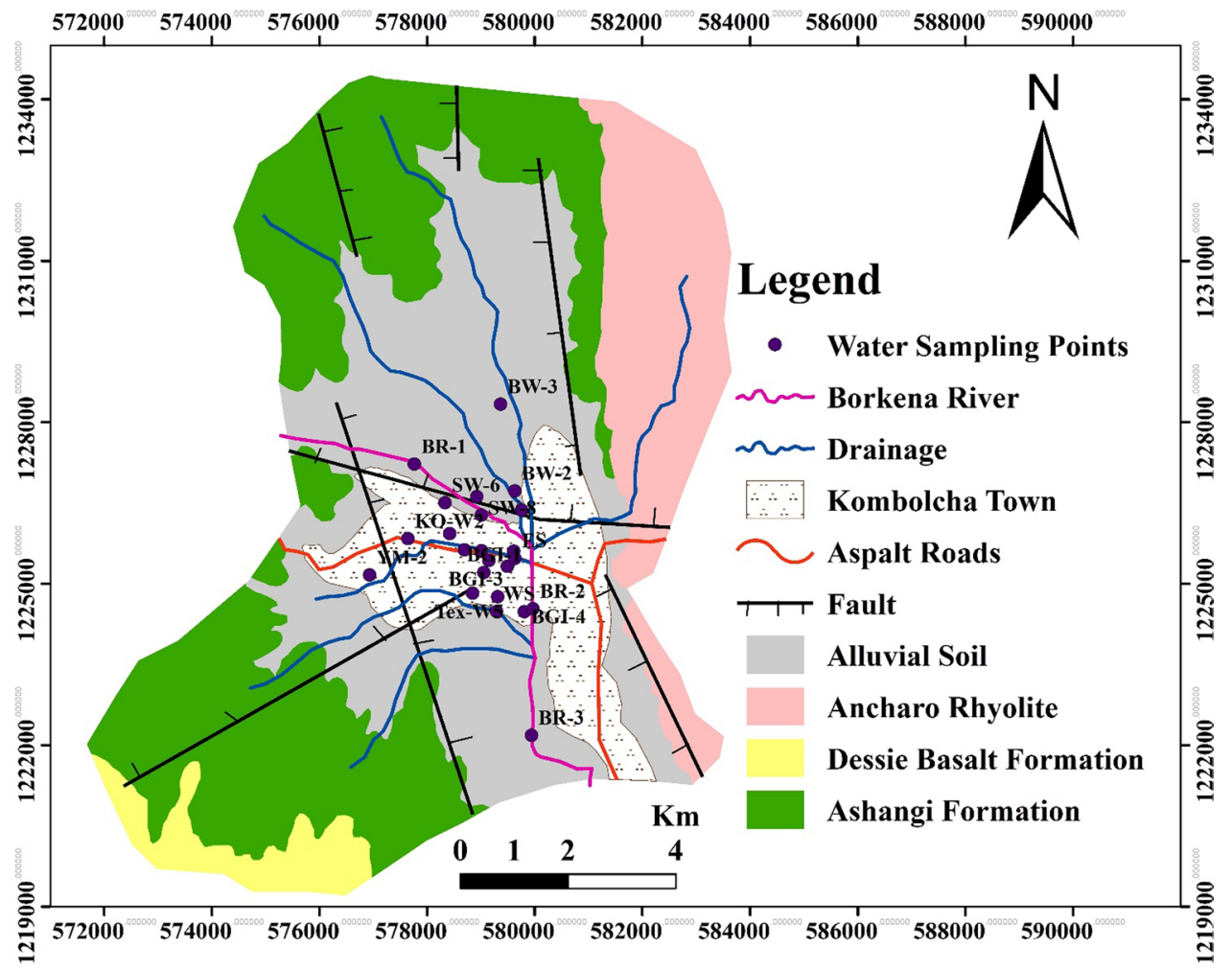

flat plains. The unit is unconformably overlain by Dessie basalt formation. Ashangie basalt is found in N and NW, and SW of the highlands of the study area. The Dessie basalt formation is exposed in the western plateau area forming a chain of ridges like Tossa Mountain, cliffs along the escarpment and river cuts (Wolfenden 2003; Tesfaye et al. 2010). The contact with the underlying Ashangie basalt is marked by about 20 -m-thick plagioclase phyric basalt marked by 
50-cm-thick paleosoil from top and bottom as seen along Dessie-Kombolcha road. This unit forms a N-S trending hill in the east part of the study area, which is named after the locality called Ancharo (Tesfaye et al. 2010). The alluvial-colluvial sediments are exposed in the low land plain and the central part of the study and cover about $45 \%$ of the mapped area. They are represented by black cotton and reddish-brown silty to sandy soil with few outcrops of diatomite. The black cotton soil is commonly seen along the northern part of the study area around the Kombolcha Airport of the basins. The thickness of the soil is more than $3 \mathrm{~m}$ as observed along the Borkena River cut and other streams cuts. The alluvial-colluvial sediments deposited at the valley of the town Kombolcha are characterized by clay, sand, gravel, pebbles and boulders derived mainly from the escarpments (Fig. 2).

Tectonic events that led to the growth of the Rift System control the geological structure of the area. The marginal grabens are slightly elongated depressions bounded on both sides by normal faults facing each other, followed in N-S direction by most of the faults in the study region (Jacques 2018) (Fig. 2). The eastern and western ridges bounding the valley area are characterized by a system of opposite dipping faults oriented parallel to the plateau escarpments (GSE 2010) (Fig. 2).

The alluvial deposits show alternating layers of sands, gravel and clay implying that there are multiple layer aquifers. The alluvial sediment is heterogeneous and their porosity and permeability making them a very good aquifer in the study area. According to pumping test data available the existing wells in the area showed average yield $10 \mathrm{l} / \mathrm{s}$ with average transmissivity $210 \mathrm{~m}^{2} /$ day (Abraham and Assay 2011). The scoracious basalt is highly porous due to its abundant vesicles and secondary structures such as joints and fractures interconnection of vesicles (Abraham and Assay 2011).

\section{Materials and methods}

Streams such as Eyole and Werka, and Borkena River have been used to obtain surface water samples, and wells have been used to collect groundwater samples that were opened by government and private firms and hotels. Hence, a total of eighteen (18) groundwater and five (5) surface water samples were collected during the year 2017 in the dry (May) and wet seasons (December).

The water samples were collected in polyethylene bottles (250-ml plastic bottles), sample containers were washed three times with the sample solution and rinsed three times with distilled water. The containers were sealed and returned to the laboratory, and then, water samples with containers were stored in a refrigerator without freezing to minimize volatilization and biodegradation until analysis at Mekelle University geochemical laboratory (Clesceri et al. 1998).

Water samples were analyzed in Mekelle University, School of Earth Science, geochemical laboratory for major cations and anions. $\mathrm{Na}, \mathrm{K}, \mathrm{Ca}, \mathrm{Mg}, \mathrm{Fe}$ and $\mathrm{Cu}$ were analyzed using the Atomic Absorption Spectrometer. 5Ob, and $\mathrm{Cl}, \mathrm{NO}_{3}, \mathrm{NO}_{2}, \mathrm{SO}_{4}, \mathrm{PO}_{4}, \mathrm{NH} 4$ and $\mathrm{F}^{-}$were determined by UV-Vis Spectrophotometer Lambda EZ 201 (Double Beam). $\mathrm{P}^{\mathrm{H}}$, electrical conductivity and total dissolved solid were determined using $\mathrm{pH}$ meter 3310 JENWAY, Conductivity meter 4310 JENWAY and Multi-meter 3910, respectively, and total hardness of the water samples was determined by EDTA and concentration of $\mathrm{Ca}$ and $\mathrm{Mg}$. Titration method with an indicator of methyl orange and titrant $0.1 \mathrm{~N} \mathrm{HCl}$ was used to determine bicarbonate and alkalinity (APHA 1989). The credibility of the chemical analyses was assessed through calculation of electrical neutrality between cations and anions, whereby the ionic balances were within $\pm 5 \%$ (Hem 1989) and were calculated using Eq. 1 (Freeze and Cherry 1979).

$\mathrm{EN}=\frac{\sum \text { Cations }-\sum \text { Anions }}{\sum \text { Cations }+\sum \text { Anions }}$

AquaChem version 4 program was used to draw the Wilcox diagram. Statistical descriptive analysis and correlation between different parameters of water chemistry were done by Statistical Package for Social Sciences (SPSS) software package version 20.

Drinking water quality of the current study was evaluated using the water quality index (WQI) method. In this study, the values of WQI for all groundwater samples have been computed using the parameters that included EC, TDS, $\mathrm{HCO}_{3}{ }^{-}, \mathrm{Na}^{+}, \mathrm{Ca}^{2+}, \mathrm{Mg}^{2+}, \mathrm{K}^{+}, \mathrm{Cl}^{-}, \mathrm{SO}_{4}{ }^{2-}, \mathrm{NO}_{3}{ }^{-}, \mathrm{PO}_{4}{ }^{3-}, \mathrm{Cu}$, $\mathrm{Fe}$ and $\mathrm{F}$ in both dry and wet season (Table 1). The computation of WQI was carried out in three stages.

First, weights have been provided to the most significant parameters anticipated to play a significant part in deteriorating the general quality of human consumption water. Accordingly, parameters $\mathrm{NO}^{3-}, \mathrm{F}^{-}, \mathrm{SO}_{4}{ }^{2-}$ and $\mathrm{PO}_{4}{ }^{3-}$ are essential in the evaluation of water quality and the maximum weight of five has been allocated; parameters such as EC, TDS, $\mathrm{Cl}^{-}$and $\mathrm{Fe}$ weight of four have been allocated; parameters $\mathrm{Na}^{+}$and $\mathrm{HCO}_{3}$ were assigned three weights; parameters $\mathrm{Ca}^{2+}, \mathrm{Mg}^{2+}, \mathrm{K}^{+}$and $\mathrm{Cu}$ were assigned two weights depending on their significance for drinking purpose (Khanoranga 2019).

Second, for each sample the relative weight (Wi) is calculated using the following equation:

$W_{i}=\frac{w_{i}}{\sum_{i=1}^{n} w_{i}}$ 
Table 1 Assignment of relative weight to the studied groundwater quality parameters and WHO standards used for WQI calculation [explanation: EC $(\mu \mathrm{S} / \mathrm{cm})$ and all concentrations $(\mathrm{mg} / \mathrm{l})$ ]

\begin{tabular}{llll}
\hline Parameter & $\begin{array}{l}\text { WHO stand- } \\
\text { ard (2011) }\end{array}$ & Weight $\left(w_{i}\right)$ & Relative weight $\left(W_{i}\right)$ \\
\hline $\mathrm{EC}$ & 1000 & 4 & 0.08 \\
$\mathrm{TDS}$ & 500 & 5 & 0.10 \\
$\mathrm{HCO}_{3}{ }^{-}$ & 500 & 1 & 0.02 \\
$\mathrm{Na}^{+}$ & 200 & 4 & 0.08 \\
$\mathrm{Ca}^{2+}$ & 75 & 3 & 0.06 \\
$\mathrm{Mg}^{2+}$ & 50 & 3 & 0.06 \\
$\mathrm{~K}^{+}$ & 12 & 2 & 0.04 \\
$\mathrm{Cl}^{-}$ & 250 & 5 & 0.10 \\
$\mathrm{SO}_{4}{ }^{2-}$ & 250 & 5 & 0.10 \\
$\mathrm{NO}_{3}{ }^{-}$ & 50 & 5 & 0.10 \\
$\mathrm{PO}_{4}{ }^{3-}$ & 5 & 1 & 0.02 \\
$\mathrm{Cu}^{-}$ & 2 & 2 & 0.04 \\
$\mathrm{Fe}^{-}$ & 0.3 & 4 & 0.08 \\
$\mathrm{~F}^{-}$ & 1.50 & 5 & 0.10 \\
& & $\sum w_{i}=49$ & $\sum W_{i}=1$ \\
\hline
\end{tabular}

where $W_{i}$ is the relative weight, $w_{i}$ is the weight of each parameter, and $n$ is the number of parameters selected.

Third, for each parameter, a quality rating scale $\left(q_{i}\right)$ is allocated by dividing its concentration in each water sample by its corresponding standard following the guidelines set out in the WHO (2011) and multiplying the outcome by 100 :

$q_{i}=\left[\frac{C_{i}}{S_{i}}\right] \times 100$

where the $q_{i}$ is the quality rating, $C_{i}$ is the concentration of each chemical parameter in each water sample given in a unit of $\mathrm{mg} / \mathrm{l}$, and $S_{i}$ is the WHO standard for each chemical parameter in $\mathrm{mg} / \mathrm{l}$ according to the guidelines of the WHO (2011).

Finally, SI was calculated for each water quality parameter by multiplying relative weight $\left(W_{i}\right)$ with a quality rating scale $\left(q_{i}\right)$. The SI amount is equivalent to the index of water quality.

$\mathrm{SI}_{i}=W_{i} \times q_{i}$

where the $\mathrm{SI}_{i}$ is the sub-index of $i$ th parameter, $q_{i}$ is the rating based on concentration of $i$ th parameter, and $\mathrm{n}$ is the number of parameters.

$\mathrm{WQI}=\sum \mathrm{SI}_{i}$

Appropriateness of water quality for agricultural purposes was assessed utilizing the well-known irrigation water quality indicator such as sodium adsorption ratio (SAR), residual sodium carbonate (RSC), permeability index (PI), magnesium ratio (MR), Kelley's index (KI) and potential salinity (PS) (Table 2).

\section{Results and discussion}

The summarized chemical and physical parameters of the laboratory result of the surface water and groundwater for the dry and wet seasons of the study area are presented in Tables 2 and 3, respectively.

\section{Surface water and groundwater chemistry}

The mean water temperature, $\mathrm{pH}$, TDS and $\mathrm{EC}$ values of the surface waters were $28.64{ }^{\circ} \mathrm{C}, 7.72,462 \mathrm{mg} / \mathrm{l}$ and $758 \mu \mathrm{S} / \mathrm{cm}$ in the dry season and $22.28^{\circ} \mathrm{C}, 6.74,316.67 \mathrm{mg} / \mathrm{l}$ and $744 \mu \mathrm{S} / \mathrm{cm}$ in the wet season, respectively. However, groundwater samples had $27.16{ }^{\circ} \mathrm{C}, 7.36,316.67 \mathrm{mg} / \mathrm{l}$ and $519.44 \mu \mathrm{S} / \mathrm{cm}$ in the dry season and $25.09^{\circ} \mathrm{C}, 6.68$, $336.11 \mathrm{mg} / \mathrm{l}$ and $531.11 \mu \mathrm{S} / \mathrm{cm}$, respectively. The $\mathrm{pH}$ values of all waters of the study area elaborate a tendency of basic reaction among the groundwater system (Tables 2 and 3). The high value of TDS in water may originate from natural sources of rock-water interaction, factory and sewage

Table 2 Summary of water quality indices for irrigation

\begin{tabular}{ll}
\hline Indices & Formula \\
\hline Sodium adsorption ratio (SAR), Richards (1954) & $\mathrm{SAR}=\frac{\mathrm{Na}^{+}}{\sqrt{\frac{\mathrm{Ca}^{2+}+\mathrm{Mg}^{2+}}{2}}}$ \\
Residual sodium carbonate (RSC), Eaton (1950) and Richards (1954) & $\mathrm{RSC}=\left(\mathrm{CO}_{3}^{2-}+\mathrm{HCO}_{3}^{-}\right)-\left(\mathrm{Ca}^{2+}+\mathrm{Mg}^{2+}\right)$ \\
Magnesium ratio (MR), Raghunath (1987) & $\mathrm{MR}=\left[\frac{\mathrm{Mg}^{2+}}{\mathrm{Mg}^{2+}+\mathrm{Ca}^{2+}}\right] \times 100$ \\
Kelly's index (KI), Kelly (1963) & $\mathrm{KI}=\frac{\mathrm{Na}^{+}}{\mathrm{Mg}^{2+}+\mathrm{Ca}^{2+}}$ \\
Potential salinity (PS), Doneen (1964) & $\mathrm{PS}=\mathrm{Cl}^{-}+\frac{1}{2} \mathrm{SO}_{4}^{2-}$ \\
Permeability index (PI), Doneen (1964) & $\mathrm{PI}=\left[\frac{\mathrm{Na}^{+}+\mathrm{HCO}_{3}^{-}}{\mathrm{Ca}^{2+}+\mathrm{Mg}^{2+}+\mathrm{Na}^{+}}\right] \times 100$ \\
\hline
\end{tabular}

All concentrations were expressed in meq/l 
Table 3 Results of chemical analyses of the surface water and groundwater samples of the study area (date of sampling: May 2017); explanation: EC $(\mu \mathrm{S} / \mathrm{cm})$, concentrations $(\mathrm{mg} / \mathrm{l})$, temperature $\left({ }^{\circ} \mathrm{C}\right)$

\begin{tabular}{|c|c|c|c|c|c|c|c|c|c|c|c|}
\hline No & Sample Id & $\mathrm{T}$ & $\mathrm{pH}$ & TDS & $\mathrm{EC}$ & $\mathrm{Ca}^{2+}$ & $\mathrm{Mg}^{2+}$ & $\mathrm{K}^{+}$ & $\mathrm{Na}^{+}$ & $\mathrm{NH}_{4}^{+}$ & $\mathrm{Cl}^{-}$ \\
\hline \multicolumn{12}{|c|}{ Surface water } \\
\hline 1 & BR-1 & 30 & 8.42 & 346.57 & 486 & 46 & 7 & 0.67 & 15 & 0.001 & 6.89 \\
\hline 2 & BR-2 & 32.5 & 7.04 & 624.68 & 876 & 137 & 7 & 1.01 & 35 & 0.005 & 25.62 \\
\hline 3 & BR-3 & 26 & 8.35 & 464.95 & 652 & 73 & 8 & 0.92 & 32 & 0.005 & 24.69 \\
\hline 4 & ES & 26.5 & 7.72 & 631.82 & 886 & 102 & 7 & 1.01 & 51 & 0.007 & 41.72 \\
\hline \multirow[t]{5}{*}{5} & WS & 28.2 & 7.76 & 1369.17 & 1920 & 182 & 5 & 1.07 & 147 & 0.008 & 39.51 \\
\hline & Min & 26 & 7 & 346.57 & 486 & 46 & 5 & 0.67 & 15 & 0.001 & 6.89 \\
\hline & Max & 32.5 & 8.4 & 1369.2 & 1920 & 182 & 8 & 1.07 & 147 & 0.008 & 41.72 \\
\hline & SD & 2.67 & 0.56 & 399.21 & 560 & 53.4 & 1.1 & 0.16 & 52.4 & 0.003 & 14 \\
\hline & Mean & 28.64 & 7.86 & 687.44 & 964 & 108 & 6.8 & 0.936 & 56 & 0.005 & 27.69 \\
\hline \multicolumn{12}{|c|}{ Groundwater } \\
\hline 6 & BW-1 & 24.7 & 7.98 & 490.62 & 688 & 59 & 8 & 0.81 & 30 & 0.002 & 21.62 \\
\hline 7 & BW-2 & 24.6 & 8.01 & 418.59 & 587 & 49 & 6 & 0.73 & 36 & 0.003 & 23.83 \\
\hline 8 & BW-3 & 24.3 & 7.23 & 488.48 & 685 & 103 & 9 & 1.05 & 17 & 0.006 & 12.18 \\
\hline 9 & BGI-1 & 29.2 & 8.04 & 301.65 & 423 & 55 & 5 & 0.73 & 31 & 0.003 & 24.07 \\
\hline 10 & BGI-3 & 28.2 & 8.27 & 250.3 & 351 & 29 & 4 & 0.29 & 21 & 0.003 & 22.85 \\
\hline 11 & BGI-4 & 28.1 & 8.32 & 258.86 & 363 & 26 & 4 & 0.52 & 38 & 0.002 & 21.11 \\
\hline 12 & ELF-W1 & 25 & 7.51 & 657.77 & 921 & 175 & 10 & 1.07 & 42 & 0.001 & 38.67 \\
\hline 13 & SSH-W & 25.9 & 7.21 & 730.94 & 1025 & 109 & 11 & 1.07 & 25 & 0.009 & 22.78 \\
\hline 14 & SW-6 & 26.6 & 7.46 & 430.00 & 603 & 85 & 7 & 0.98 & 32 & 0.004 & 24.15 \\
\hline 15 & SW-8 & 25.4 & 8.06 & 358.69 & 503 & 77 & 6 & 0.51 & 31 & 0.002 & 23.52 \\
\hline 16 & Tex-W2 & 28.1 & 6.93 & 448.55 & 629 & 85 & 4 & 0.91 & 41 & 0.002 & 31.53 \\
\hline 17 & Tex-W5 & 27 & 7.78 & 360.83 & 506 & 42 & 5 & 0.91 & 30 & 0.002 & 25.14 \\
\hline 18 & Tex-W9 & 28.4 & 8.28 & 233.90 & 328 & 36 & 4 & 0.62 & 32 & 0.005 & 21.73 \\
\hline 19 & Tex-W10 & 35.4 & 8.28 & 270.27 & 379 & 36 & 4 & 0.68 & 31 & 0.002 & 25.13 \\
\hline 20 & Tan-W & 24.5 & 7.72 & 759.46 & 1065 & 168 & 10 & 1.09 & 32 & 0.006 & 26.17 \\
\hline 21 & KCTW-2R & 27.5 & 7.95 & 343.72 & 482 & 76 & 6 & 0.69 & 25 & 0.004 & 19.87 \\
\hline 22 & KO-W2 & 27.9 & 7.59 & 733.08 & 1028 & 106 & 8 & 1.01 & 52 & 0.006 & 25.23 \\
\hline \multirow[t]{5}{*}{23} & YM-2 & 28.1 & 7.94 & 298.08 & 418 & 38 & 4 & 0.93 & 29 & 0.001 & 22.11 \\
\hline & Min & 24.3 & 6.93 & 233.90 & 328 & 26 & 4 & 0.29 & 17 & 0.001 & 12.18 \\
\hline & Max & 35.4 & 8.32 & 759.46 & 1065 & 175 & 11 & 1.09 & 52 & 0.009 & 38.67 \\
\hline & SD & 2.61 & 0.41 & 176.01 & 247 & 44.13 & 2.4 & 0.23 & 8.03 & 0.002 & 5.22 \\
\hline & Mean & 27.16 & 7.81 & 435.21 & 610 & 75.22 & 6.39 & 0.81 & 31.9 & 0.004 & 23.98 \\
\hline No & Sample Id & $\mathrm{SO}_{4}^{2-}$ & $\mathrm{HCO}_{3}^{-}$ & $\mathrm{CO}_{3}^{-}$ & $\mathrm{NO}_{3}^{-}$ & $\mathrm{NO}_{2}^{-}$ & $\mathrm{PO}_{4}{ }^{3-}$ & $\mathrm{F}^{-}$ & $\mathrm{Fe}$ & $\mathrm{Cu}$ & $\%$ Errol \\
\hline \multicolumn{12}{|c|}{ Surface water } \\
\hline 1 & BR-1 & 75 & 115.84 & 2.92 & 3.61 & 0.03 & 0.003 & 0.002 & 0.31 & 0.42 & -3.6 \\
\hline 2 & BR-2 & 132.51 & 304.66 & 0.164 & 8.17 & 0.05 & 0.007 & 0.002 & 0.47 & 0.42 & 2.05 \\
\hline 3 & BR-3 & 82.81 & 198.81 & 3.29 & 12.32 & 0.05 & 0.005 & 0.003 & 0.51 & 0.43 & -2.26 \\
\hline 4 & ES & 151.73 & 230.23 & 1.11 & 23.64 & 0.06 & 0.008 & 0.005 & 0.69 & 0.51 & -3.7 \\
\hline \multirow[t]{5}{*}{5} & WS & 169.53 & 673.5 & 3.04 & 15.26 & 0.09 & 0.009 & 0.009 & 0.73 & 0.73 & -0.32 \\
\hline & Min & 75 & 115.84 & 0.164 & 3.61 & 0.03 & 0.003 & 0.002 & 0.31 & 0.42 & \\
\hline & Max & 169.53 & 673.5 & 3.29 & 23.64 & 0.09 & 0.009 & 0.009 & 0.73 & 0.73 & \\
\hline & SD & 41.8 & 217 & 1.39 & 7.57 & 0.022 & 0.002 & 0.003 & 0.17 & 0.13 & \\
\hline & Mean & 122.32 & 304.61 & 2.1 & 12.6 & 0.056 & 0.006 & 0.004 & 0.54 & 0.50 & \\
\hline \multicolumn{12}{|c|}{ Groundwater } \\
\hline 6 & BW-1 & 105.00 & 119.19 & 1.47 & 12.33 & 0.05 & 0.004 & 0.003 & 0.49 & 0.51 & -0.65 \\
\hline 7 & BW-2 & 103.37 & 104.22 & 1.46 & 13.42 & 0.04 & 0.002 & 0.005 & 0.62 & 0.63 & -2.9 \\
\hline 8 & BW-3 & 141.63 & 208.01 & 0.341 & 6.29 & 0.04 & 0.003 & 0.001 & 0.67 & 0.45 & -1.17 \\
\hline
\end{tabular}


Table 3 (continued)

\begin{tabular}{|c|c|c|c|c|c|c|c|c|c|c|c|}
\hline No & Sample Id & $\mathrm{SO}_{4}^{2-}$ & $\mathrm{HCO}_{3}^{-}$ & $\mathrm{CO}_{3}^{-}$ & $\mathrm{NO}_{3}^{-}$ & $\mathrm{NO}_{2}^{-}$ & $\mathrm{PO}_{4}{ }^{3-}$ & $\mathrm{F}^{-}$ & $\mathrm{Fe}$ & $\mathrm{Cu}$ & $\%$ Error \\
\hline 9 & BGI-1 & 69.63 & 123.24 & 1.2 & 18.16 & 0.03 & 0.001 & 0.002 & 0.41 & 0.42 & 0.51 \\
\hline 10 & BGI-3 & 63.81 & 45.63 & 1.7 & 11.38 & 0.02 & 0.003 & 0.001 & 0.46 & 0.39 & -4.62 \\
\hline 11 & BGI-4 & 81.65 & 55.34 & 1.6 & 10.57 & 0.01 & 0.002 & 0.008 & 0.31 & 0.41 & -1.96 \\
\hline 12 & ELF-W1 & 171.89 & 413.45 & 0.659 & 13.28 & 0.05 & 0.006 & 0.007 & 0.54 & 0.61 & -1.11 \\
\hline 13 & SSH-W & 76.00 & 339.46 & 0.511 & 11.35 & 0.08 & 0.009 & 0.006 & 0.73 & 0.69 & -3.36 \\
\hline 14 & SW-6 & 133.78 & 152.78 & 0.501 & 11.58 & 0.03 & 0.001 & 0.003 & 0.58 & 0.39 & 0.55 \\
\hline 15 & SW-8 & 65.52 & 185.19 & 1.33 & 16.82 & 0.02 & 0.002 & 0.001 & 0.34 & 0.51 & 2.94 \\
\hline 16 & Tex-W2 & 82.67 & 201.12 & 0.084 & 13.15 & 0.02 & 0.006 & 0.001 & 0.35 & 0.23 & 2.1 \\
\hline 17 & Tex-W5 & 31.58 & 136.78 & 0.792 & 11.53 & 0.04 & 0.002 & 0.003 & 0.31 & 0.73 & 0.23 \\
\hline 18 & Tex-W9 & 52.41 & 81.61 & 1.51 & 15.15 & 0.01 & 0.003 & 0.003 & 0.32 & 0.32 & 2.91 \\
\hline 19 & Tex-W10 & 32.58 & 107.59 & 1.57 & 12.71 & 0.03 & 0.003 & 0.001 & 0.32 & 0.22 & 1.25 \\
\hline 20 & Tan-W & 154.92 & 364.33 & 1.46 & 10.68 & 0.08 & 0.007 & 0.009 & 0.63 & 0.82 & 2.33 \\
\hline 21 & KCTW-2R & 72.74 & 159.97 & 1.14 & 12.93 & 0.04 & 0.003 & 0.001 & 0.52 & 0.48 & 4.39 \\
\hline 22 & KO-W2 & 186.72 & 228.12 & 0.629 & 11.32 & 0.07 & 0.007 & 0.007 & 0.67 & 0.62 & -1.77 \\
\hline \multirow[t]{5}{*}{23} & YM-2 & 32.54 & 129.79 & 0.986 & 9.15 & 0.03 & 0.002 & 0.004 & 0.28 & 0.57 & -1.34 \\
\hline & Min & 31.58 & 45.63 & 0.08 & 6.29 & 0.01 & 0.001 & 0.001 & 0.28 & 0.22 & \\
\hline & Max & 186.72 & 413.45 & 1.7 & 18.16 & 0.08 & 0.009 & 0.009 & 0.73 & 0.82 & \\
\hline & SD & 47.85 & 103.98 & 0.5 & 2.68 & 0.02 & 0.002 & 0.003 & 0.15 & 0.17 & \\
\hline & Mean & 92.14 & 175.32 & 1.05 & 12.32 & 0.04 & 0.004 & 0.004 & 0.48 & 0.5 & \\
\hline
\end{tabular}

discharges. The maximum TDS (1369.17 mg/l) and EC $(1920 \mu \mathrm{S} / \mathrm{cm})$ values were measured in surface water from sample number WS (Werka Stream). It had been sampled from the direct discharge liquid waste of BGI-Brewery Factory (Tables 3 and 4, and Figs. 1 and 2).

Calcium concentration ranged from 46 to 182 and 61 to $170 \mathrm{mg} / \mathrm{l}$ in the dry and wet season for surface waters, respectively, while in groundwater samples it ranged from 26 to 175 and 41 to $178 \mathrm{mg} / \mathrm{l}$ in the dry season and wet season, respectively. The average calcium concentration value of all waters was 75.22 and $70.67 \mathrm{mg} / \mathrm{l}$ for dry and wet season, respectively. The range of magnesium in surface water varies from 5 to $8 \mathrm{mg} / \mathrm{l}$ and 6 to $12 \mathrm{mg} / \mathrm{l}$ in the dry and wet season, respectively. However, the concentration in groundwater varies from 4 to $11 \mathrm{mg} / \mathrm{l}$ in the dry season and 4 to $25 \mathrm{mg} / \mathrm{l}$ in the wet season. Analysis of surface water samples shows that potassium value varies between 0.67 and $1.07 \mathrm{mg} / \mathrm{l}$ and 0.95 and 1.03 in the dry and rainy season, respectively, while water samples taken from groundwater vary from 0.29 to $1.09 \mathrm{mg} / \mathrm{l}$ in the dry season and 0.54 to $1.57 \mathrm{mg} / \mathrm{l}$ in the wet season. The higher $\mathrm{Ca}^{2+}$ content can cause abdominal ailments and is undesirable for domestic uses as it causes encrustation and scaling (Sarath Prasanth et al. 2012). The long-term agricultural activities may also directly or indirectly influence mineral dissolution in groundwater (Bohlke 2002).

The expected source of potassium in groundwater is most likely to be the $\mathrm{K}^{+}$feldspar weathering and the application of artificial fertilizers in agricultural activities. According to Kolahchi and Jalali (2006), the lower potassium concentration in groundwater is owing to its greater resistance to weathering and fixation in the form of clay minerals leading to nutrient loss. Sodium concentration in surface water varied from 15 to $147 \mathrm{mg} / \mathrm{l}$ in the dry season and 9 to $18 \mathrm{mg} / \mathrm{l}$ in the wet season, whereas in groundwater varied from 17 to $52 \mathrm{mg} / \mathrm{l}$ in the dry season and 5 to $33.11 \mathrm{mg} / \mathrm{l}$ in the wet season (Tables 3 and 4). The higher resistance of potash feldspars than sodium feldspars to chemical weathering results lower concentration of $\mathrm{K}^{+}$than $\mathrm{Na}^{+}$in the study area (Tables 3 and 4).

Relatively, the higher concentration of $\mathrm{Na}^{+}$and $\mathrm{Cl}^{-}$is observed from the groundwater sample Tan-W (a well located within the Tannery Factory), surface water WS (effluent discharged from BGI-Brewery Factory) and surface water ES sampled from Eyole stream where it gets discharge from Tannery Factory and ELFORA-Meat Processing Factory (Tables 3 and 4, Figs. 1 and 2).

Bicarbonate, sulfate and chloride concentrations in surface water ranged from 115.84 to $673.5 \mathrm{mg} / \mathrm{l}$ in the dry season and 164.7 to 357.95 in the wet season, 75 to $169.53 \mathrm{mg} / \mathrm{l}$ in the dry season and 41.85 to $178 \mathrm{mg} / \mathrm{l}$ in the wet season and 6.89 to $4.72 \mathrm{mg} / \mathrm{l}$ in the dry season and 4.52 to $16.23 \mathrm{mg} / \mathrm{l}$ wet season, respectively. The concentrations in groundwater varied from 45.63 to $413.45 \mathrm{mg} / \mathrm{l}$ in the dry season and 101.3 to $377.09 \mathrm{mg} / \mathrm{l}$ in the wet season, 31.58 to $186.72 \mathrm{mg} / \mathrm{l}$ in the dry season and 36.15 to $145.61 \mathrm{mg} / \mathrm{l}$ in the dry season and 12.1 to $838.67 \mathrm{mg} / \mathrm{l}$ in the dry season and 
Table 4 Results of chemical analyses of the surface water and groundwater samples of the study area (date of sampling: Nov 2017); explanation: EC $(\mu \mathrm{S} / \mathrm{cm})$, concentrations $(\mathrm{mg} / \mathrm{l})$, temperature $\left({ }^{\circ} \mathrm{C}\right)$

\begin{tabular}{|c|c|c|c|c|c|c|c|c|c|c|c|}
\hline No. & Sample Id & $\mathrm{T}$ & $\mathrm{pH}$ & TDS & $\mathrm{EC}$ & $\mathrm{Ca}^{2+}$ & $\mathrm{Mg}^{2+}$ & $\mathrm{K}^{+}$ & $\mathrm{Na}^{+}$ & $\mathrm{NH}_{4}^{+}$ & $\mathrm{Cl}^{-}$ \\
\hline \multicolumn{12}{|c|}{ Surface water } \\
\hline 1 & BR-1 & 22.3 & 6.51 & 348 & 488 & 84 & 6 & 0.96 & 9 & 0.004 & 4.52 \\
\hline 2 & BR-2 & 22.6 & 6.68 & 527 & 739 & 132 & 8 & 0.98 & 11 & 0.003 & 7.54 \\
\hline 3 & BR-3 & 22.7 & 6.91 & 601.9 & 844 & 103 & 11 & 0.95 & 16 & 0.003 & 9.88 \\
\hline 4 & ES & 21.4 & 6.64 & 670.3 & 940 & 170 & 12 & 1.03 & 18 & 0.005 & 16.23 \\
\hline \multirow[t]{5}{*}{5} & WS & 22.4 & 7.74 & 355.1 & 498 & 61 & 7 & 0.98 & 11 & 0.006 & 5.31 \\
\hline & Min & 21.4 & 6.51 & 348 & 488 & 61 & 6 & 0.95 & 9 & 0.003 & 4.52 \\
\hline & $\operatorname{Max}$ & 22.7 & 7.74 & 670.3 & 940 & 170 & 12 & 1.03 & 18 & 0.006 & 16.23 \\
\hline & SD & 0.52 & 0.49 & 145.1 & 203.46 & 42.46 & 2.59 & 0.03 & 3.81 & 0 & 4.7 \\
\hline & Mean & 22.28 & 6.9 & 500.5 & 701.8 & 110 & 8.8 & 0.98 & 13 & 0 & 8.7 \\
\hline \multicolumn{12}{|c|}{ Groundwater } \\
\hline 6 & BW-1 & 22.8 & 8.07 & 340.2 & 477 & 75 & 19 & 0.93 & 16 & 0.002 & 5.12 \\
\hline 7 & BW-2 & 23 & 7.66 & 413.6 & 580 & 65 & 16 & 0.98 & 12 & 0.006 & 8.77 \\
\hline 8 & BW-3 & 22.8 & 7.59 & 489.9 & 687 & 96 & 5 & 1.02 & 11 & 0.005 & 6.11 \\
\hline 9 & BGI-1 & 27.5 & 7.61 & 288.5 & 404 & 45 & 7 & 0.98 & 5 & 0.002 & 5.01 \\
\hline 10 & BGI-3 & 26.6 & 7.56 & 284.5 & 349 & 53 & 5 & 0.69 & 7 & 0.003 & 4.12 \\
\hline 11 & BGI-4 & 26.4 & 7.77 & 260.3 & 365 & 48 & 4 & 0.61 & 8 & 0.002 & 5.72 \\
\hline 12 & ELF-W1 & 23.2 & 7.53 & 634.7 & 890 & 102 & 12 & 1.02 & 32 & 0.006 & 25.67 \\
\hline 13 & SSH-W & 24.2 & 8.11 & 307.4 & 431 & 44 & 7 & 1.03 & 5.8 & 0.002 & 6.11 \\
\hline 14 & SW-6 & 24.9 & 8.08 & 309.5 & 434 & 44 & 8 & 1.03 & 6.2 & 0.002 & 5.34 \\
\hline 15 & SW-8 & 24.8 & 7.69 & 349.4 & 490 & 62 & 8 & 0.83 & 18 & 0.003 & 12.15 \\
\hline 16 & Tex-W2 & 25.6 & 7.56 & 499.9 & 701 & 88 & 12 & 0.99 & 22 & 0.005 & 16.24 \\
\hline 17 & Tex-W5 & 25.3 & 7.7 & 343.7 & 482 & 52 & 11 & 1.02 & 8 & 0.003 & 3.15 \\
\hline 18 & Tex-W9 & 27.6 & 7.68 & 233.2 & 327 & 44 & 4.3 & 0.97 & 5.8 & 0.002 & 2.41 \\
\hline 19 & Tex-W10 & 26.5 & 8.01 & 244.6 & 343 & 42 & 4 & 0.54 & 12 & 0.001 & 5.67 \\
\hline 20 & Tan-W & 23.4 & 6.99 & 836.6 & 1233 & 178 & 11 & 1.22 & 33.1 & 0.005 & 39.61 \\
\hline 21 & KCTW-2R & 25.5 & 7.9 & 305.9 & 429 & 41 & 5 & 0.98 & 5.2 & 0.001 & 5.36 \\
\hline 22 & KO-W2 & 25.4 & 7.39 & 376.5 & 528 & 72 & 13 & 0.73 & 11 & 0.005 & 9.61 \\
\hline \multirow[t]{5}{*}{23} & YM-2 & 26.2 & 7.39 & 763.3 & 1069 & 121 & 25 & 1.57 & 32 & 0.009 & 25.28 \\
\hline & Min & 22.8 & 6.99 & 233.2 & 327 & 41 & 4 & 0.54 & 5 & 0.001 & 2.41 \\
\hline & Max & 27.6 & 8.11 & 836.6 & 1233 & 178 & 25 & 1.57 & 33.1 & 0.009 & 39.61 \\
\hline & SD & 1.57 & 0.28 & 176.3 & 258.17 & 35.79 & 5.77 & 0.23 & 9.69 & 0.002 & 9.98 \\
\hline & Mean & 25.09 & 7.68 & 404.5 & 567.72 & 70.67 & 9.79 & 0.95 & 13.9 & 0.004 & 10.64 \\
\hline No. & Sample Id & $\mathrm{SO}_{4}^{2-}$ & $\mathrm{HCO}_{3}^{-}$ & $\mathrm{CO}_{3}^{-}$ & $\mathrm{NO}_{3}^{-}$ & $\mathrm{NO}_{2}^{-}$ & $\mathrm{PO}_{4}{ }^{3-}$ & $\mathrm{F}^{-}$ & $\mathrm{Fe}$ & $\mathrm{Cu}$ & $\%$ Error \\
\hline \multicolumn{12}{|c|}{ Surface water } \\
\hline 1 & BR-1 & 92.49 & 164.7 & 0.029 & 2.67 & 0.04 & 0.003 & 0.006 & 0.63 & 0.59 & 3.15 \\
\hline 2 & BR-2 & 118.15 & 262.3 & 0.537 & 4.11 & 0.06 & 0.002 & 0.004 & 0.52 & 0.61 & 4.76 \\
\hline 3 & BR-3 & 98.77 & 239.1 & 0.16 & 10.76 & 0.05 & 0.004 & 0.004 & 0.76 & 0.82 & 2.59 \\
\hline 4 & ES & 178 & 357.95 & 0.025 & 6.12 & 0.03 & 0.003 & 0.003 & 0.81 & 0.69 & 0.82 \\
\hline \multirow[t]{5}{*}{5} & WS & 41.85 & 201.79 & 0.122 & 2.56 & 0.05 & 0.002 & 0.004 & 0.52 & 0.63 & -2.88 \\
\hline & Min & 41.85 & 164.7 & 0.025 & 2.56 & 0.03 & 0.002 & 0.003 & 0.52 & 0.59 & \\
\hline & $\operatorname{Max}$ & 178 & 357.95 & 0.537 & 10.76 & 0.06 & 0.004 & 0.006 & 0.81 & 0.82 & \\
\hline & SD & 49.2 & 73.16 & 0.21 & 3.4 & 0.01 & 0 & 0.001 & 0.13 & 0.09 & \\
\hline & Mean & 105.85 & 245.17 & 0.17 & 5.24 & 0.05 & 0 & 0.004 & 0.648 & 0.668 & \\
\hline \multicolumn{12}{|c|}{ Groundwater } \\
\hline 6 & BW-1 & 105.11 & 194.13 & 0.085 & 4.23 & 0.04 & 0.002 & 0.002 & 0.51 & 0.54 & 3.85 \\
\hline 7 & BW-2 & 71.89 & 213.5 & 0.289 & 4.08 & 0.06 & 0.003 & 0.003 & 0.62 & 0.62 & -1.97 \\
\hline 8 & BW-3 & 86.22 & 250.1 & 0.055 & 3.25 & 0.03 & 0.006 & 0.003 & 0.54 & 0.49 & -3.42 \\
\hline
\end{tabular}


Table 4 (continued)

\begin{tabular}{|c|c|c|c|c|c|c|c|c|c|c|c|}
\hline No. & Sample Id & $\mathrm{SO}_{4}^{2-}$ & $\mathrm{HCO}_{3}^{-}$ & $\mathrm{CO}_{3}^{-}$ & $\mathrm{NO}_{3}^{-}$ & $\mathrm{NO}_{2}^{-}$ & $\mathrm{PO}_{4}{ }^{3-}$ & $\mathrm{F}^{-}$ & $\mathrm{Fe}$ & $\mathrm{Cu}$ & $\%$ Error \\
\hline 9 & BGI-1 & 49 & 123.61 & 0.051 & 1.51 & 0.05 & 0.001 & 0.001 & 0.69 & 0.59 & -1.95 \\
\hline 10 & BGI-3 & 49.66 & 152.3 & 0.071 & 1.02 & 0.02 & 0.001 & 0.001 & 0.31 & 0.38 & -4.1 \\
\hline 11 & BGI-4 & 36.15 & 115.9 & 0.021 & 2.01 & 0.01 & 0.001 & 0.001 & 0.25 & 0.41 & 4.14 \\
\hline 12 & ELF-W1 & 98.62 & 294.1 & 0.084 & 9.13 & 0.07 & 0.005 & 0.003 & 0.51 & 0.82 & -1.6 \\
\hline 13 & SSH-W & 50.13 & 123.59 & 0.045 & 2.01 & 0.06 & 0.003 & 0.001 & 0.68 & 0.58 & -3.51 \\
\hline 14 & SW-6 & 51 & 124.13 & 0.047 & 2.81 & 0.07 & 0.002 & 0.002 & 0.72 & 0.61 & -2.18 \\
\hline 15 & SW-8 & 51.79 & 186.69 & 0.077 & 6.42 & 0.04 & 0.003 & 0.004 & 0.45 & 0.42 & -0.28 \\
\hline 16 & Tex-W2 & 63.98 & 298.54 & 0.067 & 8.12 & 0.06 & 0.003 & 0.002 & 0.45 & 0.59 & -3.39 \\
\hline 17 & Tex-W5 & 112.78 & 101.3 & 0.027 & 2.31 & 0.04 & 0.004 & 0.002 & 0.57 & 0.53 & -3.2 \\
\hline 18 & Tex-W9 & 47 & 118.92 & 0.056 & 1.25 & 0.04 & 0.002 & 0.002 & 0.61 & 0.49 & -3.2 \\
\hline 19 & Tex-W10 & 52.18 & 106.4 & 0.038 & 3.15 & 0.02 & 0.001 & 0.001 & 0.38 & 0.29 & -1.29 \\
\hline 20 & Tan-W & 145.61 & 359.23 & 0.139 & 15.62 & 0.06 & 0.04 & 0.07 & 0.78 & 1.23 & 4.53 \\
\hline 21 & KCTW-2R & 47 & 111.87 & 0.039 & 1.98 & 0.03 & 0.001 & 0.001 & 0.61 & 0.51 & -4.98 \\
\hline 22 & KO-W2 & 65.12 & 225.7 & 0.071 & 5.05 & 0.04 & 0.002 & 0.003 & 0.39 & 0.62 & -2.3 \\
\hline \multirow[t]{5}{*}{23} & YM-2 & 105.71 & 377.09 & 0.142 & 10.37 & 0.09 & 0.005 & 0.008 & 0.89 & 0.73 & 1.44 \\
\hline & Min & 36.15 & 101.3 & 0.021 & 1.02 & 0.01 & 0.001 & 0.001 & 0.25 & 0.29 & \\
\hline & Max & 145.61 & 377.09 & 0.289 & 15.62 & 0.09 & 0.04 & 0.07 & 0.89 & 1.23 & \\
\hline & SD & 30.31 & 89.98 & 0.06 & 3.89 & 0.02 & 0.01 & 0.02 & 0.17 & 0.20 & \\
\hline & Mean & 71.61 & 193.17 & 0.08 & 4.68 & 0.05 & 0.005 & 0.01 & 0.55 & 0.58 & \\
\hline
\end{tabular}

2.41 to $39.61 \mathrm{mg} / \mathrm{l}$ in the wet season for bicarbonate, sulfate and chloride, respectively (Tables 3 and 4).

Sources of chloride in groundwater include rainwater, fertilizers and sewage water pollutants (Srinivasamoorthy et al. 2014; Sarath Prasanth et al. 2012). The presence of sulfate to the groundwater and surface water is primarily because of the dissolution of filtering waters, leaching from fertilizers and municipal waste (Singh 1994). High Cl concentration also observed from groundwater sample ELF-W1 (a well located inside ELFORA-Meat Processing Factory) and surface water sample BR-2 sampled from Borkena River which is located just down the factories (Tables 3 and 4 , Figs. 1 and 2).

The concentrations of $\mathrm{Fe}$ in various parts of the study area are tabulated in Tables 3 and 4 . The concentration of $\mathrm{Fe}$ in groundwater varied from 0.28 to $0.73 \mathrm{mg} / \mathrm{l}$ and 0.25 to $0.89 \mathrm{mg} / \mathrm{l}$ in the dry and wet season, respectively (Tables 3 and 4). Its concentration in surface water, however, is higher than groundwater and ranged from 0.31 to $0.73 \mathrm{mg} / \mathrm{l}$ and 0.52 to $0.81 \mathrm{mg} / \mathrm{l}$ in the dry season and wet season, respectively (Tables 3 and 4 ).

In most of the groundwater samples, the measured major cations and anions in dry seasons are higher than the wet season due to the rock-water interaction dominancy. During the wet season, as the water level of the groundwater rises, more dilution has occurred and lower concentration than of the dry season was measured. It is obvious that surface water is more susceptible to pollution than groundwater and high concentration of most of the major ions. TDS, $\mathrm{EC}, \mathrm{Cu}$ and
Fe were recorded (Tables 3 and 4). The source of high Fe in all water could have resulted from anthropogenic activities. Untreated effluents are the main cause of increasing some parameters.

\section{Appraisal of groundwater quality for drinking purposes}

The quality parameters of drinking water have a direct effect on human health. Getting quality water for drinking purposes becomes challenging day by day due to the disturbance by anthropogenic activities.

The groundwater samples from the study were evaluated using the most desirable limit as well as the maximum permissible limit proposed by WHO (2011) (Table 1) for drinking usages.

Out of the 18 groundwater samples of the study area, the TDS value 4 of them (22.2\%) in the dry season and 3 $(16.7 \%)$ in the wet season were above the maximum permissible limit as recommended by WHO (2011) (Tables 1, 3 and 4$)$. In the $3(16.7 \%)$ in the dry season and $2(11.1 \%)$ in the wet season groundwater samples of the study area the EC values of the well are above the maximum permissible limit as recommended by WHO (2011). Variations of EC have mainly resulted from a geochemical process such as rock-water interaction, silicate weathering, ion exchange, reverse exchange and oxidation and reduction of sulfate (Ramesh 2008). 
Table 5 Water quality index (WQI) of groundwater samples of the study area

\begin{tabular}{|c|c|c|c|c|}
\hline \multirow[t]{2}{*}{ Sample ID } & \multicolumn{2}{|l|}{ May 2017} & \multicolumn{2}{|l|}{ Nov 2017} \\
\hline & WQI value & Water quality & WQI value & Water quality \\
\hline BW-1 & 45.49 & Excellent & 41.4 & Excellent \\
\hline BW-2 & 46.33 & Excellent & 44.42 & Excellent \\
\hline BW-3 & 53.69 & Good & 46.02 & Excellent \\
\hline BGI-1 & 36.29 & Excellent & 26.49 & Excellent \\
\hline BGI-3 & 31.15 & Excellent & 37.13 & Excellent \\
\hline BGI-4 & 28.49 & Excellent & 23.47 & Excellent \\
\hline ELF-W1 & 67.51 & Good & 55.38 & Good \\
\hline SSH-W & 63.96 & Good & 37.73 & Excellent \\
\hline SW-6 & 49.17 & Excellent & 39.26 & Excellent \\
\hline SW-8 & 38.01 & Excellent & 36 & Excellent \\
\hline Tex-W2 & 41.87 & Excellent & 45.43 & Excellent \\
\hline Tex-W5 & 32.24 & Excellent & 39.44 & Excellent \\
\hline Tex-W9 & 28.23 & Excellent & 32.48 & Excellent \\
\hline Tex-W10 & 28.09 & Excellent & 26.76 & Excellent \\
\hline Tan-W & 70.75 & Good & 81.22 & Good \\
\hline KCTW-2R & 41.35 & Excellent & 34.89 & Excellent \\
\hline KO-W2 & 66.89 & Good & 37.06 & Excellent \\
\hline YM-2 & 28.03 & Excellent & 73.85 & Good \\
\hline Min & 28.03 & & 23.47 & \\
\hline $\operatorname{Max}$ & 70.75 & & 81.22 & \\
\hline SD & 14.80 & & 15.03 & \\
\hline Mean & 44.31 & & 42.14 & \\
\hline
\end{tabular}

The concentration of the major ions $\left(\mathrm{Na}^{+}, \mathrm{Mg}^{2+}, \mathrm{K}^{+}, \mathrm{Cl}^{-}\right.$, $\mathrm{SO}_{4}{ }^{2-}$ and $\left.\mathrm{HCO}_{3}{ }^{-}\right), \mathrm{NO}_{3}{ }^{-}, \mathrm{PO}_{4}{ }^{3-}, \mathrm{F}^{-}$and $\mathrm{Cu}$ of the groundwater samples of the two seasons was fallen within the recommended limit for human consumption. However, in case of the concentration of $\mathrm{Ca}^{2+} 11(61.1 \%)$ of the groundwater samples were suitable for drinking applications (Tables 1, 3 and 4).

It is found from the study that all the groundwater samples were exceeded the standard levels of Fe of the World Health Organization (WHO) (Tables 1, 3 and 4). These higher amounts of $\mathrm{Fe}$ found in the study area may be harmful to the people who are using these water sources for their daily drinking purposes.

Groundwater quality for drinking of the current study was evaluated by the water quality index (WQI) method. The WQI is considerably used to evaluate the quality of water in various countries of the world (Alobaidy et al. 2010; Aghazadeh and Mogaddam 2010; Keesari et al. 2016).

The groundwater samples' calculated WQI value ranged from 28.03 to 70.75 in the dry season with an average value of 44.31 and from 23.47 to 81.22 in the wet season with an average value of 42.14 (Table 5). The calculated WQI values showed that the study area's groundwater is categorized into two water types, excellent water and good water for drinking. Thus, among all groundwater samples, the proportion of WQI classifications in the dry season was excellent $(72.2 \%)$
Table 6 Range and classification of WQI for drinking purpose in the present study

\begin{tabular}{|c|c|c|c|c|c|}
\hline \multirow[t]{2}{*}{ WQI value } & \multirow[t]{2}{*}{ Water quality } & \multicolumn{2}{|c|}{ Dry season (May 2017) } & \multicolumn{2}{|c|}{ Wet season (Nov 2017) } \\
\hline & & \# of samples & $\%$ of samples & \# of samples & $\%$ of samples \\
\hline$<50$ & Excellent water & 13 & 72.2 & 15 & 83.3 \\
\hline $50-100$ & Good water & 5 & 27.8 & 3 & 16.7 \\
\hline $100-200$ & Poor water & Nil & Nil & Nil & Nil \\
\hline $200-300$ & Very poor water & Nil & Nil & Nil & Nil \\
\hline$>300$ & $\begin{array}{l}\text { Unsuitable for } \\
\text { drinking water }\end{array}$ & Nil & Nil & Nil & Nil \\
\hline
\end{tabular}

Fig. 3 The proportion of groundwater samples in the study area classified based on WQI: a dry season, $\mathbf{b}$ wet season (a)

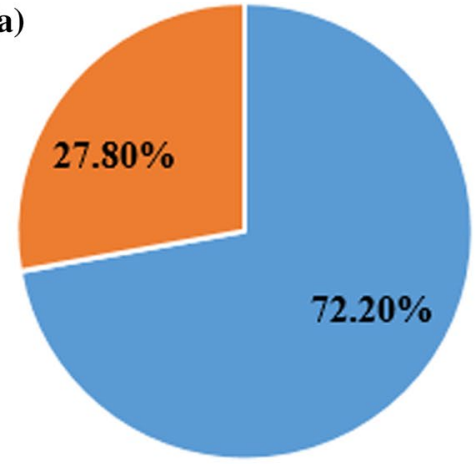

- Excellent Water " Good Water (b)

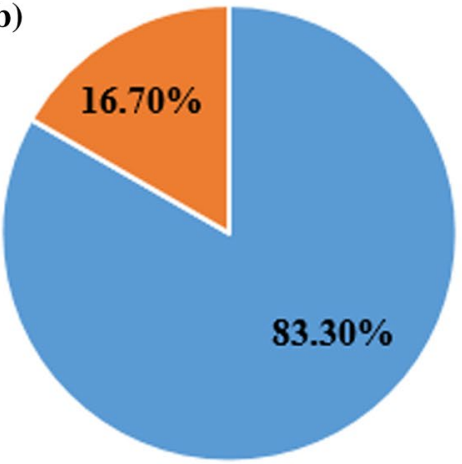

Excellent Water $\approx$ Good Water 
and good (27.8\%), while excellent (83.3\%) and good (16.7\%) in the wet season (Table 6 and Fig. 3).

Comparing the two seasons, water in the wet seasons has a better quality than dry seasons. This may be resulted from the fewer concentration components in the wet season owing to greater water levels in the wells during the wet season than in the dry season (Tables 5 and 6 and Fig. 3). Variation in some of the physicochemical parameters may be also due to anthropogenic and geogenic sources (Khanoranga 2018).

\section{Appraisal of groundwater and surface water quality for irrigation purposes}

The use of poor irrigation water quality reduces agricultural crop yields. The mineral composition denoted by the quality of irrigational water and the quality proves its effects on plants and soil. It is clear that high-quality and quantity crops yield from the usage of high-quality water.

Groundwater pumped from wells and source water for irrigation purposes may comprise substantial chemical constituents derived from natural processes and human activities that may reduce crop yield and deteriorate soil fertility (Jalali 2009). The chemical composition of irrigation water directly or indirectly impacts crop production by influencing nutrient availability in plants. Irrigation water chemistry varies with the source, local and regional geology. For example, soil water with an elevated salt concentration can be extremely damaging to plants by altering metabolic processes, retarding plant growth. The awareness of irrigation water quality is serious to understand what management changes are necessary for long-standing productivity (Jalali 2011).
Parameters such as sodium adsorption ratio (SAR), residual sodium carbonate (RSC), magnesium ratio (MR), permeability index (PI) and electrical conductivity (EC) were used to evaluate irrigation water quality for two seasons. These parameters are crucial for irrigation purposes in the assessment of groundwater and surface water (Tables 2 and 8).

\section{Sodium adsorption ratio (SAR)}

Sodium adsorption ratio is the most commonly used parameter to assess the effects of exchangeable sodium on the physical condition of the soil (Richards 1954). The excess sodium in water reacts with soil that changes the composition of the soil and reduces the permeability of the soil. Then, the soil becomes more impermeable and compressed. Richards (1954) categorized irrigation water as suitable based into four groups based on sodium adsorption ratio SAR (Table 8). The graph of sodium hazard versus salinity hazard (Wilcox 1955) shows that the entire water samples gathered during dry and wet seasons fall into categories C2-S1 and C3-S1, showing low alkaline risks and outstanding irrigation water (Fig. 4).

The Wilcox (1955) sodium hazard versus salinity hazard graph demonstrates that all water samples collected during dry and wet seasons fall into C2-S1 and C3-S1 classifications, demonstrating small alkaline hazards and great irrigation water (Fig. 4).

During the dry season (May 2017), the SAR value of the groundwater sample ranges from 0.43 to 1.83 with an average of 1.04 and the rainy season (November 2017) ranges from 0.18 to 0.80 with an average of 0.39 (Table 7). According to the Richards (1954) classification, all groundwater samples have very low SAR values in both seasons showing that all water samples in the study area are in an excellent category (Table 8). The SAR value of the surface water

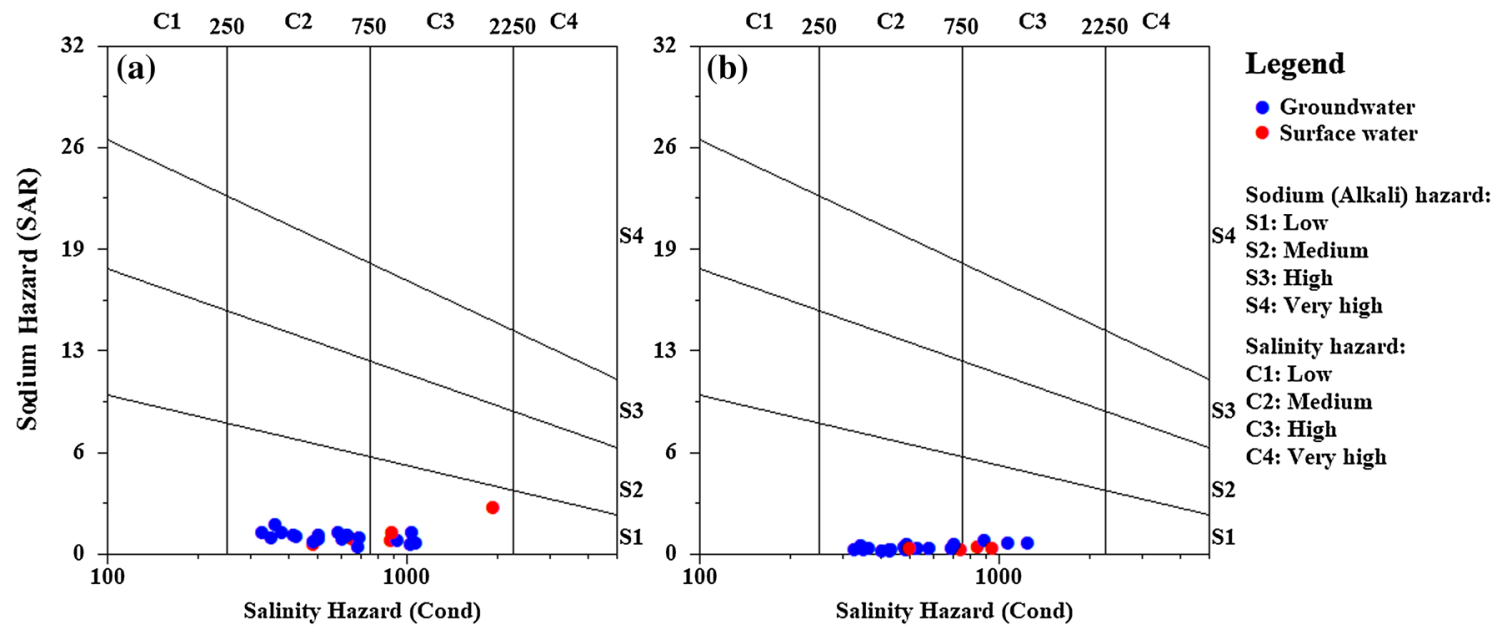

Fig. 4 Classification of water samples based on salinity and sodium hazard: $\mathbf{a}$ dry season and $\mathbf{b}$ wet season 
Table 7 Results of water quality indices for irrigation

\begin{tabular}{|c|c|c|c|c|c|c|c|c|}
\hline \multirow[t]{2}{*}{ No. } & \multirow[t]{2}{*}{ Sample Id } & \multicolumn{7}{|c|}{ Dry season (May 2017) } \\
\hline & & SAR & PI & RSC & PS & MR & KI & $\mathrm{EC}$ \\
\hline \multicolumn{9}{|c|}{ Surface water } \\
\hline 1 & BR-1 & 0.54 & 57.53 & -0.88 & 0.98 & 20.03 & 0.23 & 486 \\
\hline 2 & BR-2 & 0.79 & 41.98 & -2.43 & 2.10 & 7.76 & 0.20 & 876 \\
\hline 3 & BR-3 & 0.95 & 56.08 & -0.94 & 1.56 & 15.28 & 0.32 & 652 \\
\hline 4 & ES & 1.32 & 52.70 & -1.87 & 2.76 & 10.15 & 0.39 & 886 \\
\hline \multirow[t]{5}{*}{5} & WS & 2.93 & 61.08 & 1.63 & 2.88 & 4.33 & 0.67 & 1920 \\
\hline & Min & 0.54 & 41.98 & -2.43 & 0.98 & 4.33 & 0.20 & 486 \\
\hline & Max & 2.93 & 61.08 & 1.63 & 2.88 & 20.03 & 0.67 & 1920 \\
\hline & SD & 0.95 & 7.30 & 1.56 & 0.80 & 6.21 & 0.19 & 559.8 \\
\hline & Mean & 1.31 & 53.87 & -0.90 & 2.06 & 11.51 & 0.36 & 964 \\
\hline \multicolumn{9}{|c|}{ Groundwater } \\
\hline 6 & BW-1 & 0.97 & 55 & -1.61 & 1.70 & 18.25 & 0.36 & 688 \\
\hline 7 & BW-2 & 1.29 & 63.7 & -1.19 & 1.75 & 16.78 & 0.53 & 587 \\
\hline 8 & BW-3 & 0.43 & 39 & -2.47 & 1.82 & 12.57 & 0.13 & 685 \\
\hline 9 & BGI-1 & 1.07 & 61.41 & -1.10 & 1.40 & 13.02 & 0.43 & 423 \\
\hline 10 & BGI-3 & 0.97 & 66.03 & -0.97 & 1.31 & 18.50 & 0.51 & 351 \\
\hline 11 & BGI-4 & 1.83 & 79.37 & -0.67 & 1.45 & 20.21 & 1.01 & 363 \\
\hline 12 & ELF-W1 & 0.83 & 38.85 & -2.78 & 2.88 & 8.60 & 0.19 & 921 \\
\hline 13 & SSH-W & 0.61 & 46.3 & -0.78 & 1.43 & 14.25 & 0.17 & 1025 \\
\hline 14 & SW-6 & 0.90 & 47.83 & -2.31 & 2.07 & 11.94 & 0.29 & 603 \\
\hline 15 & SW-8 & 0.91 & 54.29 & -1.26 & 1.35 & 11.37 & 0.31 & 503 \\
\hline 16 & Tex-W2 & 1.18 & 56.56 & -1.28 & 1.75 & 7.19 & 0.39 & 629 \\
\hline 17 & Tex-W5 & 1.16 & 73.42 & -0.24 & 1.04 & 16.39 & 0.52 & 506 \\
\hline 18 & Tex-W9 & 1.35 & 72.37 & -0.74 & 1.16 & 15.46 & 0.65 & 328 \\
\hline 19 & Tex-W10 & 1.31 & 76.95 & -0.31 & 1.05 & 15.46 & 0.63 & 379 \\
\hline 20 & Tan-W & 0.65 & 36.13 & -3.20 & 2.35 & 8.92 & 0.15 & 1065 \\
\hline 21 & KCTW-2R & 0.74 & 50.29 & -1.63 & 1.32 & 11.50 & 0.25 & 482 \\
\hline 22 & KO-W2 & 1.31 & 51.03 & -2.20 & 2.66 & 11.05 & 0.38 & 1028 \\
\hline \multirow[t]{5}{*}{23} & YM-2 & 1.19 & 77.91 & -0.07 & 0.96 & 14.77 & 0.57 & 418 \\
\hline & Min & 0.43 & 36.13 & -3.20 & 0.96 & 7.19 & 0.13 & 328 \\
\hline & Max & 1.83 & 79.37 & -0.07 & 2.88 & 20.21 & 1.01 & 1065 \\
\hline & SD & 0.33 & 14.03 & 0.90 & 0.55 & 3.63 & 0.22 & 246.7 \\
\hline & Mean & 1.04 & 58.14 & -1.38 & 1.64 & 13.68 & 0.42 & 610.2 \\
\hline \multirow[t]{2}{*}{ No. } & Sample Id & \multicolumn{7}{|c|}{ Wet season (Nov 2017) } \\
\hline & & SAR & PI & RSC & PS & MR & KI & $\mathrm{EC}$ \\
\hline \multicolumn{9}{|c|}{ Surface water } \\
\hline 1 & BR-1 & 0.26 & 40 & -1.99 & 1.09 & 10.52 & 0.08 & 488 \\
\hline 2 & BR-2 & 0.25 & 33 & -2.94 & 1.44 & 9.07 & 0.07 & 739 \\
\hline 3 & BR-3 & 0.40 & 39.6 & -2.13 & 1.31 & 14.95 & 0.11 & 844 \\
\hline 4 & ES & 0.36 & 31.2 & -3.62 & 2.31 & 10.41 & 0.08 & 940 \\
\hline \multirow[t]{5}{*}{5} & WS & 0.36 & 56 & -0.32 & 0.59 & 15.89 & 0.13 & 498 \\
\hline & Min & 0.25 & 31.2 & -3.62 & 0.59 & 9.07 & 0.07 & 488 \\
\hline & $\operatorname{Max}$ & 0.40 & 56 & -0.32 & 2.31 & 15.89 & 0.13 & 940 \\
\hline & SD & 0.07 & 9.77 & 1.24 & 0.63 & 3.04 & 0.03 & 203 \\
\hline & Mean & 0.34 & 40 & -2.20 & 1.35 & 12.17 & 0.09 & 702 \\
\hline \multicolumn{9}{|c|}{ Groundwater } \\
\hline 6 & BW-1 & 0.43 & 41.3 & -2.13 & 1.24 & 29.43 & 0.13 & 477 \\
\hline
\end{tabular}


Table 7 (continued)

\begin{tabular}{|c|c|c|c|c|c|c|c|c|}
\hline \multirow[t]{2}{*}{ No. } & \multirow[t]{2}{*}{ Sample Id } & \multicolumn{7}{|c|}{ Wet season (Nov 2017) } \\
\hline & & SAR & PI & RSC & PS & MR & KI & $\mathrm{EC}$ \\
\hline 7 & BW-2 & 0.35 & 47 & -1.06 & 1.00 & 28.84 & 0.11 & 580 \\
\hline 8 & BW-3 & 0.30 & 44 & -1.11 & 1.07 & 7.90 & 0.09 & 687 \\
\hline 9 & BGI-1 & 0.18 & 53.9 & -0.80 & 0.65 & 20.39 & 0.08 & 404 \\
\hline 10 & BGI-3 & 0.25 & 56 & -0.56 & 0.63 & 13.44 & 0.10 & 349 \\
\hline 11 & BGI-4 & 0.30 & 56.1 & -0.83 & 0.54 & 12.06 & 0.13 & 365 \\
\hline 12 & ELF-W1 & 0.80 & 48 & -1.27 & 1.75 & 16.22 & 0.23 & 890 \\
\hline 13 & SSH-W & 0.21 & 55.3 & -0.75 & 0.69 & 20.75 & 0.09 & 431 \\
\hline 14 & SW-6 & 0.23 & 54.2 & -0.82 & 0.68 & 23.03 & 0.09 & 434 \\
\hline 15 & SW-8 & 0.57 & 55.8 & -0.70 & 0.88 & 17.52 & 0.21 & 490 \\
\hline 16 & Tex-W2 & 0.58 & 49.9 & -0.49 & 1.12 & 18.33 & 0.18 & 701 \\
\hline 17 & Tex-W5 & 0.26 & 42.5 & -1.84 & 1.26 & 25.83 & 0.10 & 482 \\
\hline 18 & Tex-W9 & 0.22 & 58.7 & -0.60 & 0.56 & 13.86 & 0.10 & 327 \\
\hline 19 & Tex-W10 & 0.47 & 62.4 & -0.68 & 0.70 & 13.55 & 0.21 & 343 \\
\hline 20 & Tan-W & 0.65 & 34.4 & -3.91 & 2.63 & 9.23 & 0.15 & 1233 \\
\hline 21 & KCTW-2R & 0.20 & 58.8 & -0.63 & 0.64 & 16.72 & 0.09 & 429 \\
\hline 22 & KO-W2 & 0.31 & 46.7 & -0.97 & 0.95 & 22.91 & 0.10 & 528 \\
\hline \multirow[t]{5}{*}{23} & YM-2 & 0.69 & 40.8 & -1.92 & 1.81 & 25.38 & 0.17 & 1069 \\
\hline & Min & 0.18 & 34.4 & -3.91 & 0.54 & 7.90 & 0.08 & 327 \\
\hline & Max & 0.80 & 62.4 & -0.49 & 2.63 & 29.43 & 0.23 & 1233 \\
\hline & SD & 0.19 & 7.65 & 0.84 & 0.55 & 6.40 & 0.05 & 258 \\
\hline & Mean & 0.39 & 50.3 & -1.17 & 1.04 & 18.63 & 0.13 & 568 \\
\hline
\end{tabular}

varies from 0.54 to 2.93 and 0.25 to 0.40 in the dry and wet season, respectively (Tables 7). Irrigation groundwater and surface water with a high concentration of $\mathrm{Na}^{+}$and low $\mathrm{Ca}^{2+}$ favors the presence of ion exchange by saturation of $\mathrm{Na}^{+}$and destroys the soil structure owing to the dispersion of clay particles (Todd 1980) resulting in minor production due to difficulty in cultivation (Subba Rao 2006). According to Tables 7 and 8 results, groundwater has better quality than surface water and results from the wet season have better quality than dry season in both groundwater and surface water of the study area.

\section{Electrical conductivity (EC)}

The concentration of EC is very essential for the classification of irrigation water. As measured by electrical conductivity, the salinity hazard decreases plant productivity (Johnson and Zhang 1990). The mean value of EC of groundwater is $610.2 \mu \mathrm{S} / \mathrm{cm}$ and $568 \mu \mathrm{S} / \mathrm{cm}$ in the dry and wet season, respectively. However, surface water has a mean value of $964 \mu \mathrm{S} / \mathrm{cm}$ and $702 \mu \mathrm{S} / \mathrm{cm}$ during the dry season and rainy season, respectively (Table 7). To classify irrigation water as low, the complete concentration of soluble salts in irrigation water can be demonstrated. Most of the groundwater and surface water samples in the area fall under good and permissible for irrigation purposes (Wilcox 1955) in both seasons (Table 8). The highest value of EC in groundwater and surface water was measured in the wet season, and in general, surface water has high electrical conductivity than groundwater (Table 7).

\section{Residual sodium carbonate (RSC)}

The calculated RSC was used to assess the dangerous impact of carbonate and bicarbonate concentration on water quality for agricultural usages in the water sample (Aghazadeh and Mogaddam 2010). Higher $\mathrm{HCO}_{3}{ }^{-}$concentration of in water outcomes precipitation of $\mathrm{Ca}^{2+}$ and $\mathrm{Mg}^{2+}$ as $\mathrm{CO}_{3}{ }^{2-}$. However, in the study area is geologically composed of basaltic type rocks which are composed of alkali feldspars (non calcareous aquifers). At some stage of the water-rock interaction substantial dissolution of sodium silicate is made by adding of $\mathrm{CO}^{2}$ to the waters and the reaction of carbonic acid with feldspar minerals in the presence of water releases $\mathrm{HCO}_{3}$. Thus the high carbonate minerals $\left(\mathrm{HCO}_{3}-\right)$ in both seasons is resulted from silicate weathering (Chidambaram et al. 2012; Rogers 1989; Elango et al. 2003). When the $\mathrm{CO}_{3}{ }^{2-}+\mathrm{HCO}_{3}{ }^{-}$concentration exceeds the $\mathrm{Ca}^{2+}+\mathrm{Mg}^{2+}$ concentration, it will affect the adequacy of irrigation water.

Aghazadeh and Mogaddam (2010), based on RSC values, categorized irrigation water into three classifications (Table 8). Table 7 indicates that the computed RSC values 


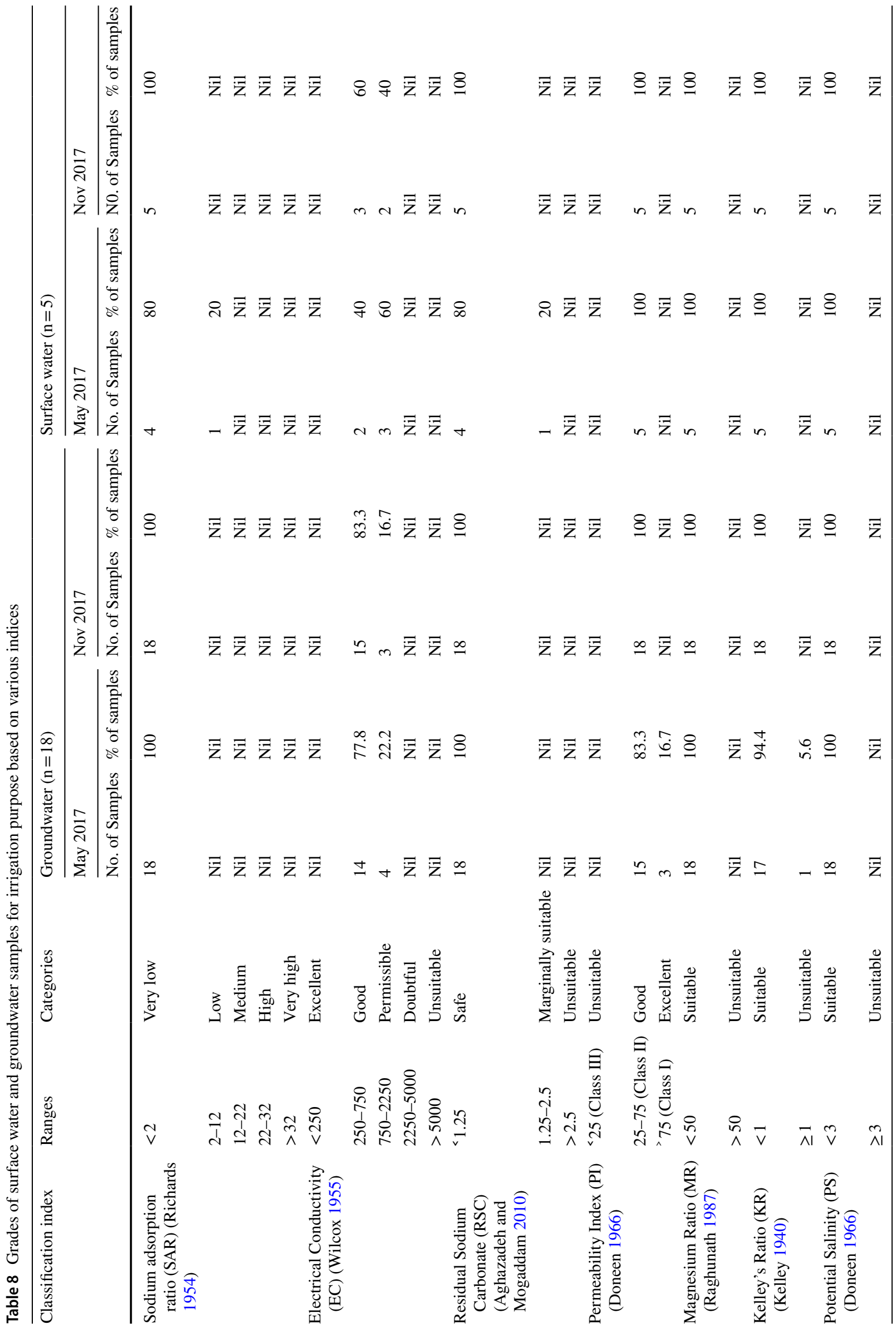


for groundwater samples range from -3.2 to -0.07 and a mean value of $-1.38 \mathrm{meq} / \mathrm{l}$ and from -3.91 to -0.49 and a mean value of $-1.17 \mathrm{meq} / \mathrm{l}$ in the dry and wet season, respectively. The calculated $\mathrm{RSC}$ values for surface water for the two seasons range from -2.43 to 1.63 and a mean value of $-0.90 \mathrm{meq} / \mathrm{l}$ and from -3.62 to -0.32 and a mean value of $-2.20 \mathrm{meq} / \mathrm{l}$ in the dry and wet season, respectively. In both seasons, the mean value of groundwater is lower than surface water revealing groundwater better quality than the surface water of the study area (Table 7).

According to Table 8, except for one surface water sample in the dry season, all groundwater and surface water samples of the study area are categorized into the safe quality for irrigation.

\section{Permeability index (PI)}

The content of sodium, calcium, magnesium and bicarbonate affects soil permeability, and the long-term use of irrigation water affects it. The study area varies from 36.13 to 79.37 and 34.4 to 62.4 during the dry and wet seasons, respectively, and surface water samples range from 41.98 to 61.08 and 31.2 to 56.0 during the dry and wet seasons, respectively (Tables 7). Regarding PI values, three groundwater samples showed class III (excellent) in the dry season. However, the rest groundwater samples and all surface water samples in the study area fall under class II (good) in both seasons (Tables 8).

\section{Magnesium ratio (MR)}

The excess quantity of magnesium in water affects soil quality, which can lead to a decrease in crop yields (Joshi et al. 2009). In the dry season and wet season, the calculated magnesium risk values from the research area's surface water vary from 4.33 to 20.03 and 9.07 to 15.89 , respectively, and groundwater ranges from 7.12 to 20.21 and 7.90 to 29.43 , respectively (Table 7 ). In total, $100 \%$ of the analyzed groundwater and surface water samples are appropriate for irrigation exercise in both seasons based on the Raghunath (1987) classification (Table 8).

\section{Kelly's index (KI)}

The Kelly index is also an important parameter measured against sodium ion levels in meq/l against magnesium and calcium ion for irrigation water quality. The statistical analysis of the Kelly index of the present study's groundwater samples ranges from 0.20 to 0.67 and 0.07 to 0.13 during the dry and wet seasons, respectively, and surface water samples vary from 41.98 to 61.08 and 31.2 to 56.0 during the dry and wet seasons, respectively (Tables 7). According to Kelley (1940) classification, except for one groundwater sample, all groundwater and surface water samples fall into the suitable category in both seasons and are appropriate for irrigation (Table 8).

\section{Potential salinity (PS)}

Groundwater samples' prospective salinity has been categorized into 3 classes (Doneen 1966) (Table 8). The study area's calculated potential salinity (PS) for groundwater varied from 0.96 to 2.88 and 0.54 to 2.63 in the dry and wet season, respectively. Surface water ranged from 0.98 to 2.88 and 0.59 to 2.31 in the dry and wet season, respectively (Tables 7). The mean values of PS in the surface water are greater than groundwater presenting surface water is more polluted than groundwater of the study area (Table 7). However, according to the Doneen (1966) classification, 100\% of groundwater and surface water samples are appropriate for irrigation exercise. But wet season results showed better quality (Table 8).

In general, the calculated and evaluated irrigation parameters for groundwater samples are low as compared to surface waters. This is because the surface waters in the study area were polluted by the untreated industrial and municipal wastes. This can be supported, for example, by sample Id of WS and ES, where the highest value of irrigation parameters (SAR, PI, RSC, etc.) was recorded (Table 7). Surface water WS was sampled from Werka Stream, and ES was from Eyole Stream where each stream gets a direct untreated discharge from BGI-Brewery Factory and Tannery factory, respectively (Figs. 1 and 2). Comparing the two seasons, groundwater's in the wet season is better than in the dry season for irrigation purposes. This is because the concentration of major ions in groundwater is higher in the dry season than in the wet season (Tables 3, 4 and 7).

\section{Conclusions}

Assessment of surface water and groundwater samples during the wet and dry seasons indicated by higher concentrations of constituents during the dry season, including $\mathrm{Ca}^{2+}$, $\mathrm{Na}^{+}, \mathrm{K}^{+}, \mathrm{Mg}^{2+}, \mathrm{HCO}_{3}{ }^{-}, \mathrm{SO}_{4}^{2}, \mathrm{Cl}^{-}$and TDS. The maximum concentration of ions, electrical conductivity and total dissolved solids measured and/or analyzed in surface water were sample number WS (Werka Stream). It was sampled from a direct discharge from a factory nearby.

WQI of the current study was computed by using eighteen groundwater sampling points and fourteen parameters (EC, TDS, HCO3, $\mathrm{Na}, \mathrm{Ca}, \mathrm{Mg}, \mathrm{K}, \mathrm{Cl}, \mathrm{SO}_{4}{ }^{2-}$, NO3, PO4, $\mathrm{Cu}, \mathrm{Fe}$ and $\mathrm{F}$ ) to evaluate the suitability of water for drinking purposes. According to WQI, groundwater samples of the present study show excellent $(72.8 \%)$ and good $(27.8 \%)$ in the dry season and excellent (83.3\%) and good $(16.7 \%)$

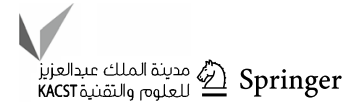


in the wet season. It is seen that wet seasons are of better quality than dry seasons. This can explain the decrease in concentration of elements in the wet season because the water level in the wells is higher in the wet season than in the dry season.

TDS, EC and $\mathrm{Ca}^{2+}$ concentration values of some of the groundwater samples and all for Fe concentrations are above the maximum permissible limit for drinking as recommended by WHO (2011). However, regarding the $\mathrm{Na}^{+}$, $\mathrm{Mg}^{2+}, \mathrm{K}^{+}, \mathrm{Cl}^{-}, \mathrm{SO}_{4}{ }^{2-}, \mathrm{HCO}_{3}{ }^{-}, \mathrm{NO}_{3}{ }^{-}, \mathrm{PO}_{4}{ }^{3-}, \mathrm{F}^{-}$and $\mathrm{Cu}$ concentrations of the groundwater samples of the two seasons were fallen within the recommended limit for human consumption.

To evaluate the groundwater and surface water for irrigation uses SAR, RSC, MR, PI, KI and EC were used to evaluate irrigation water quality for two seasons. The results reveal that most of the groundwater and surface water samples were suitable for irrigation with some places in the study locations that belong to the good and permissible. However, none of them are unsuitable for irrigation.

According to Table 8, $100 \%$ of the calculated values of MR and PS for all groundwater and surface water sample, all SAR and RSC except for one surface water samples and all KI except for one groundwater samples are suitable for irrigational practice in both seasons.

The value of the electrical conductivity of water samples of the study area samples is falling into the good and permissible in both dry and wet seasons for agricultural usages. Graph of sodium hazard versus salinity hazard the entire water sample collected in May and November 2017 falls into category $\mathrm{C} 2-\mathrm{S} 1$ and $\mathrm{C} 3-\mathrm{S} 1$, showing low alkali hazards and waters are grouped under excellent irrigation water.

In general, as the head of the groundwater increases the concentration of most ions decreases, and hence, the concentrations of most ions in the wet season are lower than in the dry season. This leads that waters in wet seasons are more suitable than in the dry season for drinking and irrigation purposes. Effluents from the different factories and industries of the area are the main cause for pollution of groundwater and surface water.

Acknowledgements This work was supported by Wollo University Research Project (WU/125/2017), and the author is grateful to Wollo University for its valuable financial support. The author is grateful for the constructive comments and suggestions of anonymous reviewers for improving the manuscript.

Open Access This article is licensed under a Creative Commons Attribution 4.0 International License, which permits use, sharing, adaptation, distribution and reproduction in any medium or format, as long as you give appropriate credit to the original author(s) and the source, provide a link to the Creative Commons licence, and indicate if changes were made. The images or other third party material in this article are included in the article's Creative Commons licence, unless indicated otherwise in a credit line to the material. If material is not included in the article's Creative Commons licence and your intended use is not permitted by statutory regulation or exceeds the permitted use, you will need to obtain permission directly from the copyright holder. To view a copy of this licence, visit http://creativecommons.org/licenses/by/4.0/.

\section{References}

Abraham M, Assay G (2011) Groundwater resource assessment for water point selection in Kombolcha town (Kombolcha Textile Factory compound and its surroundings). Unpublished report, Wollo University

Aghazadeh N, Mogaddam AA (2010) Assessment of groundwater quality and its suitability for drinking and agricultural uses in the Oshnavieharea, Northwest of Iran. J Environ Prot 1:30-40

Alobaidy AHMJ, Abid HS, Maulood BK (2010) Application of water quality index for assessment of Dokan lake ecosystem, Kurdistan region, Iraq. J Water Resour Prot 2(9):792-798

APHA (1989) Standard methods for the examination of water and wastewater, 17th edn. American Public Health Association, Washington, p 1268

Bohlke JK (2002) Groundwater recharge and agricultural contamination. Hydrogeol J 10:153-179

Brown RM, Mc Clelland N, Deininger RA, Tozer RG (1970) A water quality index — do we dare. Water Sewage Works 117:339-343

Chandra S, Singh PK, Tiwari AK, Panigrahy BP, Kumar A (2015) Evaluation of hydrogeological factors and their relationship with seasonal water table fluctuation in Dhanbad district, Jharkhand, India. ISH J Hydraul Eng 21(2):193-206

Chidambaram S, Anandhan P, Prasanna MV, Srinivasamoorthy K, Vasanthavigar M (2012) Major ion chemistry and identification of hydrogeochemical processes controlling groundwater in and around Neyveli Lignite Mines, Tamil Nadu, South India. Arab J Geosci. https://doi.org/10.1007/s12517-012-0589-3

Clesceri LC, Greenberg AE, Eaton AD (1998) Standard methods for the Examination of water and waste water, 20th edn. American Health Association, Baltimore

Cloutier V, Lefebvre Rene, Therrien Rene, Savard Martine M (2008) Multivariate statistical analysis of geochemical data as indicative of the hydrogeochemical evolution of groundwater in a sedimentary rock aquifer system. J Hydrol 353:294-313

Connor R (2015) The United Nations world water development report 2015: water for a sustainable world (vol 1). UNESCO Publishing, Paris

Das Brijraj K, Kaur P (2007) Geochemistry of surface and subsurface waters of Rewalsar Lake, Mandi district, Himachal Pradesh: constraints on weathering and erosion. J Geol Soc India 69(5):1020-1030

Deininger RA, Maciunas JJ (1971) A water quality of environmental and industrial health, school of public health. University of Michigan, Ann Arbor

Doneen LD (1964) Notes on water quality in agriculture published as a water science and engineering paper 4001. Department of Water Science and Engineering, University of California

Doneen LD (1966) Water quality requirement for agriculture. In: Proceedings of the national symposium quality standard for natural waters. University of Michigan, annual report, 213-218

Eaton FM (1950) Significance of carbonate irrigation water. Soils Sci 69(2):123-133

Elango L, Rannan R, Senthil KM (2003) Major ion chemistry and identification of hydrogeochemical processes of groundwater in 
a part of Kancheepuram district, Tamil Nadu, India. J Environ Geosci 10:157-166

Freeze RA, Cherry JA (1979) Groundwater, vol 7632. Prentice-Hall Inc., Englewood Cliffs, p 604

GSE (2010) Geology geochemistry and gravity survey of the Dessie Area, Memoir 28

Hamere Y, Eyasu E (2017) Contamination of rivers and water reservoirs in and around Addis Ababa City and actions to combat it. Environ Pollut Clim Change 1:116

Hem JD (1989) Study and interpretation of chemical characteristics of natural waters, 3rd edn. US Geological Survey Water Supply Paper 2254

Horton RK (1965) An index number system for rating water quality. J Water Pollut Control Fed 37:300-305

Jacques V (2018) Geology of afar (East Africa), regional geology reviews. Springer, Cham

Jalali M (2009) Phosphorous concentration, solubility and species in the groundwater in a semi-arid basin, southern Malayer, western Iran. Environ Geol 57:1011-1020

Jalali M (2011) Hydrogeochemistry of groundwater and its suitability for drinking and agricultural use in Nahavand, Western Iran. Nat Resour Res 20:1. https://doi.org/10.1007/s11053-010-9131-z

Johnson G, Zhang H (1990) Classification of irrigation water quality. Oklahoma cooperative extension fact sheets. Available online at http://www.osuextra.com

Joshi DM, Kumar A, Agrawal N (2009) Assessment of the irrigation water quality of river Ganga in Haridwar District India. J Chem 2(2):285-292

Keesari T, Sinha UK, Deodhar A, Krishna SH, Ansari A, Mohokar H, Dash A (2016) High fluoride in groundwater of an industrialized area of Eastern India (Odisha): inferences from geochemical and isotopic investigation. Environ Earth Sci 75(14):1-17

Kelley WP (1940) Permissible composition and concentration of irrigation waters. ASCE, Proc, p 66

Kelly WP (1963) Use of saline irrigation water. Soil Sci 95:355-391

Khanoranga KS (2018) An assessment of groundwater quality for irrigation and drinking purposes around brick kilns in three districts of Balochistan province, Pakistan, through water quality index and multivariate statistical approaches. J Geochem Explor. https://doi. org/10.1016/j.gexplo.2018.11.007

Khanoranga KS (2019) An assessment of groundwater quality for irrigation and drinking purposes around brick kilns in three districts of Balochistan province, Pakistan, through water quality index and multivariate statistical approaches. J Geochem Explor 197:14-26

Kolahchi Z, Jalali M (2006) Simulating leaching of potassium in a sandy soil using simple and complex models. Agric Water Manag 85:85-94. https://doi.org/10.1016/j.agwat.2006.03.011

Li P, Qian H, Wu J (2010) Groundwater quality assessment based on improved water quality index in Pengyang County, Ningxia, Northwest China. E-J Chem 7(S1):S209-S216. https://doi. org/10.1155/2010/451304

Mengesha T, Tadiwos C, Werkneh H (1996) Explanation of the geological map of Ethiopia, Ethiopian institute of geological surveys. Addis Ababa 3:79

Mohammed A (2015) Assessment of heavy metal contamination levels of the vegetables cultivated along two tributaries of Borkena River at Kombolcha Town. Msc Thesis, Addis Ababa University

Pati S, Dash MK, Mukherjee CK, Dash B, Pokhrel S (2014) Assessment of water quality using multivariate statistical techniques in the coastal region of Visakhapatnam, India. Environ Monit Assess 186:6385-6402. https://doi.org/10.1007/s10661-014-3862-y
Raghunath HM (1987) Groundwater. Wiley Eastern Ltd., New Delhi, pp 344-369

Rajesh R, Sreedhara Murthy TR, Raghavan BR (2002) The utility of multivariate statistical techniques in hydrogeochemical studies: an example from Karnataka, India. Water Res 36:2437-2442

Ramesh K (2008) Hydrochemical studies and effect of irrigation on groundwater quality Tondiar basin Tamilnadu. Unpublished Ph.D thesis, Anna University, Chennai, Tamilnadu

Richards LA (1954) Diagnosis and improvement of saline and alkali soils. Agricultural handbook no.60. United States Department of Agriculture, Washington DC

Rogers RJ (1989) Geochemical comparison of groundwater in areas of New England, NewYork, and Pennsylvania. Groundwater 27:690-712

Sarath Prasanth SV, Magesh NS, Jitheshlal KV, Chandrasekar N, Gangadhar K (2012) Evaluation of groundwater quality and its suitability for drinking and agricultural use in the coastal stretch of Alappuzha District, Kerala, India. Appl Water Sci 2:165-175

Singh KP (1994) Temporal changes in the chemical quality of ground water in Ludhiana area. Curr Sci 66:375-378

Srinivasamoorthy K, Gopinath M, Chidambaram S, Vasanthavigar M, Sarma VS (2014) Hydrochemical characterization and quality appraisal of groundwater from Pungar sub basin, Tamilnadu, India. J King Saud Univ Sci 26:37-52

Subba Rao N (2006) Seasonal variation of groundwater quality in a part of Guntur district, Andhra Pradesh, India. Environ Geol 49:413-429

Tesfaye D, Genet Y, Abraham M, Yibeltal T, Yonas T, Getachew B, Mohammed E and Meran W (2010) Geology geochemistry and gravity survey of the dessie area. Un published report, pp 68

Tiwari JN, Manzoor A (1988) Water quality index for Indian rivers. In: Trivedy RK (ed) Ecology and pollution of Indian rivers. Aashish Publishing House, New Delhi, pp 271-286

Todd DK (1980) Ground water hydrology. Wiley, New York

Van der Gun J (2012) Groundwater and global change: trends, opportunities and challenges. UNESCO Side Publication Series 01, UNESCO, Paris

Varol S, Davraz A (2015) Evaluation of the groundwater quality with WQI (water quality index) and multivariate analysis: a case study of the Tefenni plain (Burdur/Turkey). Environ Earth Sci. https:// doi.org/10.1007/s12665-014-3531-z

Verma DK, Bhunia GS, Shit PK, Kumar S, Mandal J, Padbhushan R (2016) Spatial variability of groundwater quality of Sabour block, Bhagalpur district (Bihar, India). Appl Water Sci. https:// doi.org/10.1007/s13201-016-0380-9

WHO (2011) Guideline for drinking water quality, 4th edn. World Health Organization, Geneva

Wilcox LV (1955) Classification and use of irrigation waters. U.S. Department of Agriculture, Washington D.C. Circular No. 969

Wolfenden E (2003) Evolution of the southern Red Sea Rift: birth of a magmatic margin. Unpublished Ph.D. thesis, Royal Holloway, University of London, 237

Publisher's Note Springer Nature remains neutral with regard to jurisdictional claims in published maps and institutional affiliations. 